\title{
SOLUTIONS FORMULAS FOR SOME GENERAL SYSTEMS OF DIFFERENCE EQUATIONS
}

\author{
Y. AKROUR, M. KARA, N. TOUAFEK, AND Y. YAZLIK
}

Received 31 May, 2020

Abstract. In this paper, we give explicit formulas of the solutions of the two general systems of difference equations

$$
\begin{aligned}
& x_{n+1}=f^{-1}\left(a g\left(y_{n}\right)+b f\left(x_{n-1}\right)+c g\left(y_{n-2}\right)+d f\left(x_{n-3}\right)\right), \\
& y_{n+1}=g^{-1}\left(a f\left(x_{n}\right)+b g\left(y_{n-1}\right)+c f\left(x_{n-2}\right)+d g\left(y_{n-3}\right)\right),
\end{aligned}
$$

and

$$
\begin{aligned}
& x_{n+1}=f^{-1}\left(a+\frac{b}{g\left(y_{n}\right)}+\frac{c}{g\left(y_{n}\right) f\left(x_{n-1}\right)}+\frac{d}{g\left(y_{n}\right) f\left(x_{n-1}\right) g\left(y_{n-2}\right)}\right), \\
& y_{n+1}=g^{-1}\left(a+\frac{b}{f\left(x_{n}\right)}+\frac{c}{f\left(x_{n}\right) g\left(y_{n-1}\right)}+\frac{d}{f\left(x_{n}\right) g\left(y_{n-1}\right) f\left(x_{n-2}\right)}\right),
\end{aligned}
$$

where $n \in \mathbb{N}_{0}, f, g: D \longrightarrow \mathbb{R}$ are " $1-1$ " continuous functions on $D \subseteq \mathbb{R}$, the initial values $x_{-i}$, $y_{-i}, i=0,1,2,3$ are arbitrary real numbers in $D$ and the parameters $a, b, c$ and $d$ are arbitrary real numbers. Our results considerably extend some existing results in the literature.

2010 Mathematics Subject Classification: 39A10, 40A05

Keywords: Systems of difference equations, form of solutions, stability of equilibrium points.

\section{INTRODUCTION AND PRELIMINARIES}

Difference equations are used to describes real discrete models in various branches of modern sciences such as, biology, economy, control theory. This explain why a big number of papers is devoted to this subject, see for example ([1] - [20]). It is clear that if we want to understand our models, we need to know the behavior of the solutions of the equations of the models, and this fact will be possible if we can solve in closed form these equations. One can find in the literature a lot of works on difference equations where explicit formulas of the solutions are given, see for instance [1], [2], [5], [8], [9], [10], [7], [12], [11], [13], [16], [15], [14], [17], [18], [21], [20], [22]. Such type of difference equations and systems is called solvable difference equations. In the present work, we continue our interest in solvable difference equations, more 
precisely, we will solve the following two general systems of difference equations

$$
\begin{aligned}
& x_{n+1}=f^{-1}\left(a g\left(y_{n}\right)+b f\left(x_{n-1}\right)+c g\left(y_{n-2}\right)+d f\left(x_{n-3}\right)\right), \\
& y_{n+1}=g^{-1}\left(a f\left(x_{n}\right)+b g\left(y_{n-1}\right)+c f\left(x_{n-2}\right)+d g\left(y_{n-3}\right)\right),
\end{aligned}
$$

and

$$
\begin{aligned}
& x_{n+1}=f^{-1}\left(a+\frac{b}{g\left(y_{n}\right)}+\frac{c}{g\left(y_{n}\right) f\left(x_{n-1}\right)}+\frac{d}{g\left(y_{n}\right) f\left(x_{n-1}\right) g\left(y_{n-2}\right)}\right), \\
& y_{n+1}=g^{-1}\left(a+\frac{b}{f\left(x_{n}\right)}+\frac{c}{f\left(x_{n}\right) g\left(y_{n-1}\right)}+\frac{d}{f\left(x_{n}\right) g\left(y_{n-1}\right) f\left(x_{n-2}\right)}\right),
\end{aligned}
$$

where $n \in \mathbb{N}_{0}, f, g: D \longrightarrow \mathbb{R}$ are one to one ("1 - 1") continuous functions on $D \subseteq$ $\mathbb{R}$, the initial values $x_{-i}, y_{-i}, i=0,1,2,3$ are arbitrary real numbers in $D$ and the parameters $a, b, c$ and $d$ are arbitrary real numbers.

In our study, we are inspired and motivated by the ideas, the equations and the systems of some recent published papers. The papers, [1], [2] and especially [15] are our main motivation in the present work. The obtained results considerably generalize some existing results in the literature, see [1], [2], [3], [4], [5], [12], [11], [13], [16], [15], [14], [17], [18], [21].

Now, we will recall some known results that will be useful, in solving in closed form our systems. To this end, let consider the two following homogeneous fourth order linear difference equations

$$
\begin{aligned}
& R_{n+1}=a R_{n}+b R_{n-1}+c R_{n-2}+d R_{n-3}, n \in \mathbb{N}_{0}, \\
& S_{n+1}=-a S_{n}+b S_{n-1}-c S_{n-2}+d S_{n-3}, n \in \mathbb{N}_{0},
\end{aligned}
$$

where the initial values $R_{0}, R_{-1}, R_{-2}, R_{-3}, S_{0}, S_{-1}, S_{-2}$ and $S_{-3}$ and the parameters $a, b, c$ and $d, d \neq 0$ are real numbers. Noting that the formulas of the solutions of equations (1.1) and (1.2), see [2], are expressed in terms of the the sequence $\left(J_{n}\right)_{n=0}^{+\infty}$ defined by the recurrent relation

$$
J_{n+4}=a J_{n+3}+b J_{n+2}+c J_{n+1}+d J_{n}, \quad n \in \mathbb{N}_{0},
$$

and the special initial values

$$
J_{0}=0, \quad J_{1}=0, \quad J_{2}=1 \text { and } J_{3}=a .
$$

The closed form of the solutions of $\left(J_{n}\right)_{n=0}^{+\infty}$ and many properties of them are well known in the literature see for example [19], [6].

The following result from [2], gave the general terms of (1.1) and (1.2) in terms of the sequence $\left(J_{n}\right)_{n=0}^{+\infty}$. The obtained formulas will be very useful to obtain the formulas of the solutions of our systems.

Lemma 1. We have for all $n \in \mathbb{N}_{0}$,

$$
\begin{gathered}
R_{n}=d J_{n+1} R_{-3}+\left(c J_{n+1}+d J_{n}\right) R_{-2}+\left(J_{n+3}-a J_{n+2}\right) R_{-1}+J_{n+2} R_{0}, \\
S_{n}=(-1)^{n+1}\left[d J_{n+1} S_{-3}-\left(c J_{n+1}+d J_{n}\right) S_{-2}+\left(J_{n+3}-a J_{n+2}\right) S_{-1}-J_{n+2} S_{0}\right] .
\end{gathered}
$$


Consider the following fourth order linear system of difference equations

$u_{n+1}=a v_{n}+b u_{n-1}+c v_{n-2}+d u_{n-3}, v_{n+1}=a u_{n}+b v_{n-1}+c u_{n-2}+d v_{n-3}, n \in \mathbb{N}_{0}$,

where the initial values $u_{-i}, v_{-i}, i=0,1,2,3$ are nonzero real numbers.

In [2], the authors got the solutions of (1.7), in terms of the sequence $\left(J_{n}\right)_{n=0}^{+\infty}$ and the initial values $u_{-i}, v_{-i}, i=0,1,2,3$.

For the interest of the readers we show how we can solve system (1.7).

Lemma 2. The solutions of the system (1.7) are given by the following formulas

$$
u_{2 n-1}=d J_{2 n} u_{-3}+\left(c J_{2 n}+d J_{2 n-1}\right) v_{-2}+\left(J_{2 n+2}-a J_{2 n+1}\right) u_{-1}+J_{2 n+1} v_{0}, n \in \mathbb{N} \text {, }
$$

$u_{2 n}=d J_{2 n+1} v_{-3}+\left(c J_{2 n+1}+d J_{2 n}\right) u_{-2}+\left(J_{2 n+3}-a J_{2 n+2}\right) v_{-1}+J_{2 n+2} u_{0}, n \in \mathbb{N}_{0}$,

$v_{2 n-1}=d J_{2 n} v_{-3}+\left(c J_{2 n}+d J_{2 n-1}\right) u_{-2}+\left(J_{2 n+2}-a J_{2 n+1}\right) v_{-1}+J_{2 n+1} u_{0}, n \in \mathbb{N}$,

$v_{2 n}=d J_{2 n+1} u_{-3}+\left(c J_{2 n+1}+d J_{2 n}\right) v_{-2}+\left(J_{2 n+3}-a J_{2 n+2}\right) u_{-1}+J_{2 n+2} v_{0}, n \in \mathbb{N}_{0}$.

Proof. From (1.7), we get

$u_{n+1}+v_{n+1}=a\left(v_{n}+u_{n}\right)+b\left(u_{n-1}+v_{n-1}\right)+c\left(v_{n-2}+u_{n-2}\right)+d\left(u_{n-3}+v_{n-3}\right), n \in \mathbb{N}_{0}$ and

$u_{n+1}-v_{n+1}=a\left(v_{n}-u_{n}\right)+b\left(u_{n-1}-v_{n-1}\right)+c\left(v_{n-2}-u_{n-2}\right)+d\left(u_{n-3}-v_{n-3}\right), n \in \mathbb{N}_{0}$.

Putting

$$
R_{n}=u_{n}+v_{n}, \quad S_{n}=u_{n}-v_{n}, n \geq-3,
$$

we obtain two homogeneous linear difference equations of fourth order:

$$
R_{n+1}=a R_{n}+b R_{n-1}+c R_{n-2}+d R_{n-3}, n \in \mathbb{N}_{0},
$$

and

$$
S_{n+1}=-a S_{n}+b S_{n-1}-c S_{n-2}+d S_{n-3}, n \in \mathbb{N}_{0} .
$$

Using (1.12), we get

$$
u_{n}=\frac{1}{2}\left(R_{n}+S_{n}\right), v_{n}=\frac{1}{2}\left(R_{n}-S_{n}\right), n \geq-3 .
$$

From Lemma 1, we obtain

$$
\begin{aligned}
u_{2 n-1} & =d J_{2 n} u_{-3}+\left(c J_{2 n}+d J_{2 n-1}\right) v_{-2}+\left(J_{2 n+2}-a J_{2 n+1}\right) u_{-1}+J_{2 n+1} v_{0}, n \in \mathbb{N}, \\
u_{2 n} & =d J_{2 n+1} v_{-3}+\left(c J_{2 n+1}+d J_{2 n}\right) u_{-2}+\left(J_{2 n+3}-a J_{2 n+2}\right) v_{-1}+J_{2 n+2} u_{0}, n \in \mathbb{N}_{0}, \\
v_{2 n-1} & =d J_{2 n} v_{-3}+\left(c J_{2 n}+d J_{2 n-1}\right) u_{-2}+\left(J_{2 n+2}-a J_{2 n+1}\right) v_{-1}+J_{2 n+1} u_{0}, n \in \mathbb{N}, \\
v_{2 n} & =d J_{2 n+1} u_{-3}+\left(c J_{2 n+1}+d J_{2 n}\right) v_{-2}+\left(J_{2 n+3}-a J_{2 n+2}\right) u_{-1}+J_{2 n+2} v_{0}, n \in \mathbb{N}_{0} .
\end{aligned}
$$




\section{SOLUTIONS OF A FIRST GENERAL SYSTEM OF DIFFERENCE EQUATIONS}

In this part, we will focus our interest on our first general system of difference equations, that is the system

$$
\left\{\begin{array}{l}
x_{n+1}=f^{-1}\left(a g\left(y_{n}\right)+b f\left(x_{n-1}\right)+c g\left(y_{n-2}\right)+d f\left(x_{n-3}\right)\right), \\
y_{n+1}=g^{-1}\left(a f\left(x_{n}\right)+b g\left(y_{n-1}\right)+c f\left(x_{n-2}\right)+d g\left(y_{n-3}\right)\right),
\end{array}\right.
$$

where $n \in \mathbb{N}_{0}, f, g: D \longrightarrow \mathbb{R}$ are continuous functions, with $D=D_{f}=D_{g}$, that is $f$ and $g$ have the same domain, and it is also assumed that $f, g$ are " $1-1$ " on $D \subseteq \mathbb{R}$, the initial values $x_{-i}, y_{-i}, i=0,1,2,3$ are arbitrary real numbers in $D$ and the parameters $a, b, c$ and $d$ are arbitrary real numbers.

\subsection{Explicit formulas of solutions of system (2.1) with $d \neq 0$}

In the following result, we solve in closed form (2.1).

Definition 1. A solution $\left\{x_{n}, y_{n}\right\}_{n \geq-3}$ of system (2.1) is said to be well-defined if for all $n \in \mathbb{N}_{0}$, we have

$$
a g\left(y_{n}\right)+b f\left(x_{n-1}\right)+c g\left(y_{n-2}\right)+d f\left(x_{n-3}\right) \in D_{f^{-1}},
$$

and

$$
a f\left(x_{n}\right)+b g\left(y_{n-1}\right)+c f\left(x_{n-2}\right)+d g\left(y_{n-3}\right) \in D_{g^{-1}} .
$$

Theorem 1. Let $\left\{x_{n}, y_{n}\right\}_{n \geq-3}$ be a well-defined solution of the system (2.1), then we have the following representation

$$
\begin{aligned}
& \left\{\begin{aligned}
x_{2 n-1} & =f^{-1}\left(d J_{2 n} f\left(x_{-3}\right)+\left(c J_{2 n}+d J_{2 n-1}\right) g\left(y_{-2}\right)+\left(J_{2 n+2}-a J_{2 n+1}\right) f\left(x_{-1}\right)\right. \\
& \left.+J_{2 n+1} g\left(y_{0}\right)\right), n \in \mathbb{N}, \\
x_{2 n} & =f^{-1}\left(d J_{2 n+1} g\left(y_{-3}\right)+\left(c J_{2 n+1}+d J_{2 n}\right) f\left(x_{-2}\right)+\left(J_{2 n+3}-a J_{2 n+2}\right) g\left(y_{-1}\right)\right. \\
& \left.+J_{2 n+2} f\left(x_{0}\right)\right), n \in \mathbb{N}_{0},
\end{aligned}\right. \\
& \begin{cases}y_{2 n-1}= & g^{-1}\left(d J_{2 n} g\left(y_{-3}\right)+\left(c J_{2 n}+d J_{2 n-1}\right) f\left(x_{-2}\right)+\left(J_{2 n+2}-a J_{2 n+1}\right) g\left(y_{-1}\right)\right. \\
y_{2 n} & \left.+J_{2 n+1} f\left(x_{0}\right)\right), n \in \mathbb{N}, \\
& g^{-1}\left(d J_{2 n+1} f\left(x_{-3}\right)+\left(c J_{2 n+1}+d J_{2 n}\right) g\left(y_{-2}\right)+\left(J_{2 n+3}-a J_{2 n+2}\right) f\left(x_{-1}\right)\right.\end{cases} \\
& \left.+J_{2 n+2} g\left(y_{0}\right)\right), n \in \mathbb{N}_{0} .
\end{aligned}
$$

Proof. Since the functions $f, g$ are " $1-1$ ", then from (2.1) we get

$$
\left\{\begin{array}{l}
f\left(x_{n+1}\right)=a g\left(y_{n}\right)+b f\left(x_{n-1}\right)+c g\left(y_{n-2}\right)+d f\left(x_{n-3}\right), \\
g\left(y_{n+1}\right)=a f\left(x_{n}\right)+b g\left(y_{n-1}\right)+c f\left(x_{n-2}\right)+d g\left(y_{n-3}\right), n \in \mathbb{N}_{0} .
\end{array}\right.
$$

By the change of variables

$$
X_{n}=f\left(x_{n}\right), \quad Y_{n}=g\left(y_{n}\right), n \geq-3,
$$


system (2.4) is transformed to the following one

$$
\left\{\begin{array}{l}
X_{n+1}=a Y_{n}+b X_{n-1}+c Y_{n-2}+d X_{n-3}, \\
Y_{n+1}=a X_{n}+b Y_{n-1}+c X_{n-2}+d Y_{n-3}, n \in \mathbb{N}_{0} .
\end{array}\right.
$$

Clearly (2.6) is in the form of system (1.7), by Lemma (2), we obtain the following representation of solutions

$$
\begin{gathered}
\left\{\begin{array}{l}
X_{2 n-1}=d J_{2 n} X_{-3}+\left(c J_{2 n}+d J_{2 n-1}\right) Y_{-2}+\left(J_{2 n+2}-a J_{2 n+1}\right) X_{-1}+J_{2 n+1} Y_{0}, n \in \mathbb{N}, \\
X_{2 n}=d J_{2 n+1} Y_{-3}+\left(c J_{2 n+1}+d J_{2 n}\right) X_{-2}+\left(J_{2 n+3}-a J_{2 n+2}\right) Y_{-1}+J_{2 n+2} X_{0}, n \in \mathbb{N}_{0},
\end{array}\right. \\
\left\{\begin{array}{l}
Y_{2 n-1}=d J_{2 n} Y_{-3}+\left(c J_{2 n}+d J_{2 n-1}\right) X_{-2}+\left(J_{2 n+2}-a J_{2 n+1}\right) Y_{-1}+J_{2 n+1} X_{0}, n \in \mathbb{N}, \\
Y_{2 n}=d J_{2 n+1} X_{-3}+\left(c J_{2 n+1}+d J_{2 n}\right) Y_{-2}+\left(J_{2 n+3}-a J_{2 n+2}\right) X_{-1}+J_{2 n+2} Y_{0}, n \in \mathbb{N}_{0} .
\end{array}\right.
\end{gathered}
$$

Now, by (2.5) we get that

$$
\begin{aligned}
& \begin{cases}x_{2 n-1} & =f^{-1}\left[d J_{2 n} f\left(x_{-3}\right)+\left(c J_{2 n}+d J_{2 n-1}\right) g\left(y_{-2}\right)+\left(J_{2 n+2}-a J_{2 n+1}\right) f\left(x_{-1}\right)\right. \\
& \left.+J_{2 n+1} g\left(y_{0}\right)\right], n \in \mathbb{N}, \\
x_{2 n} & =f^{-1}\left[d J_{2 n+1} g\left(y_{-3}\right)+\left(c J_{2 n+1}+d J_{2 n}\right) f\left(x_{-2}\right)+\left(J_{2 n+3}-a J_{2 n+2}\right) g\left(y_{-1}\right)\right. \\
& \left.+J_{2 n+2} f\left(x_{0}\right)\right], n \in \mathbb{N}_{0},\end{cases} \\
& \begin{cases}y_{2 n-1} & =g^{-1}\left[d J_{2 n} g\left(y_{-3}\right)+\left(c J_{2 n}+d J_{2 n-1}\right) f\left(x_{-2}\right)+\left(J_{2 n+2}-a J_{2 n+1}\right) g\left(y_{-1}\right)\right. \\
& \left.+J_{2 n+1} f\left(x_{0}\right)\right], n \in \mathbb{N}, \\
y_{2 n} \quad & g^{-1}\left[d J_{2 n+1} f\left(x_{-3}\right)+\left(c J_{2 n+1}+d J_{2 n}\right) g\left(y_{-2}\right)+\left(J_{2 n+3}-a J_{2 n+2}\right) f\left(x_{-1}\right)\right. \\
& \left.+J_{2 n+2} g\left(y_{0}\right)\right], n \in \mathbb{N}_{0} .\end{cases}
\end{aligned}
$$

Remark 1. Moreover, if $g \equiv f$ and $y_{-i}=x_{-i}, i=\overline{0,3}$ then, the system (2.1) will be the equation

$$
x_{n+1}=f^{-1}\left(a f\left(x_{n}\right)+b f\left(x_{n-1}\right)+c f\left(x_{n-2}\right)+d f\left(x_{n-3}\right)\right)
$$

and then the representation of the well-defined solutions are given by

$$
\left\{\begin{aligned}
x_{2 n-1} & =f^{-1}\left[d J_{2 n} f\left(x_{-3}\right)+\left(c J_{2 n}+d J_{2 n-1}\right) f\left(x_{-2}\right)+\left(J_{2 n+2}-a J_{2 n+1}\right) f\left(x_{-1}\right)\right. \\
& \left.+J_{2 n+1} f\left(x_{0}\right)\right], n \in \mathbb{N} \\
& =f^{-1}\left[d J_{2 n+1} f\left(x_{-3}\right)+\left(c J_{2 n+1}+d J_{2 n}\right) f\left(x_{-2}\right)+\left(J_{2 n+3}-a J_{2 n+2}\right) f\left(x_{-1}\right)\right. \\
& \left.+J_{2 n+2} f\left(x_{0}\right)\right], n \in \mathbb{N}_{0}
\end{aligned}\right.
$$


In [15], Stevic studied the equation (2.11).

Now as applications of theorem 1 , we give the following examples.

Example 1. Let

$$
f(t)=t^{2 j+1}, g(t)=t^{2 k+1}, \quad j, k \in \mathbb{N}_{0} .
$$

Then, $D_{f}=D_{g}=\mathbb{R}$, clearly the functions $f$ and $g$ are " $1-1$ " continuous functions on $\mathbb{R}$ and the system (2.1) becomes

$$
\left\{\begin{array}{l}
x_{n+1}=\left[a y_{n}^{2 k+1}+b x_{n-1}^{2 j+1}+c y_{n-2}^{2 k+1}+d x_{n-3}^{2 j+1}\right]^{\frac{1}{2 j+1}}, \\
y_{n+1}=\left[a x_{n}^{2 j+1}+b y_{n-1}^{2 k+1}+c x_{n-2}^{2 j+1}+d y_{n-3}^{2 k+1}\right]^{\frac{1}{2 k+1}}, n \in \mathbb{N}_{0} .
\end{array}\right.
$$

Then from (2.2) and (2.3), we obtain that general solution of the equation (2.14) is

$$
\begin{aligned}
& \left\{\begin{aligned}
x_{2 n-1} & =\left[d J_{2 n} x_{-3}^{2 j+1}+\left(c J_{2 n}+d J_{2 n-1}\right) y_{-2}^{2 k+1}+\left(J_{2 n+2}-a J_{2 n+1}\right) x_{-1}^{2 j+1}\right. \\
& \left.+J_{2 n+1} y_{0}^{2 k+1}\right]^{\frac{1}{2 j+1}}, n \in \mathbb{N}, \\
x_{2 n} & =\left[d J_{2 n+1} y_{-3}^{2 k+1}+\left(c J_{2 n+1}+d J_{2 n}\right) x_{-2}^{2 j+1}+\left(J_{2 n+3}-a J_{2 n+2}\right) y_{-1}^{2 k+1}\right. \\
& \left.+J_{2 n+2} x_{0}^{2 j+1}\right]^{\frac{1}{2 j+1}}, n \in \mathbb{N}_{0},
\end{aligned}\right. \\
& \left\{\begin{aligned}
y_{2 n-1} & =\left[d J_{2 n} y_{-3}^{2 k+1}+\left(c J_{2 n}+d J_{2 n-1}\right) x_{-2}^{2 j+1}+\left(J_{2 n+2}-a J_{2 n+1}\right) y_{-1}^{2 k+1}\right. \\
& \left.+J_{2 n+1} x_{0}^{2 j+1}\right]^{\frac{1}{2 k+1}}, n \in \mathbb{N}, \\
y_{2 n} & =\left[d J_{2 n+1} x_{-3}^{2 j+1}+\left(c J_{2 n+1}+d J_{2 n}\right) y_{-2}^{2 k+1}+\left(J_{2 n+3}-a J_{2 n+2}\right) x_{-1}^{2 j+1}\right. \\
& \left.+J_{2 n+2} y_{0}^{2 k+1}\right]^{\frac{1}{2 k+1}}, n \in \mathbb{N}_{0} .
\end{aligned}\right.
\end{aligned}
$$

Example 2. Let

$$
f(t)=\frac{1}{t^{2 j+1}}, g(t)=\frac{1}{t^{2 k+1}}, \quad j, k \in \mathbb{N}_{0} .
$$

Then, $D_{f}=D_{g}=\mathbb{R}-\{0\}$, clearly the functions $f$ and $g$ are " $1-1$ " continuous functions on $\mathbb{R}-\{0\}$ and the system (2.1) becomes

$$
\left\{\begin{array}{l}
x_{n+1}=\left[\frac{a}{y_{n}^{2 k+1}}+\frac{b}{x_{n-1}^{2 j+1}}+\frac{c}{y_{n-2}^{2 k+1}}+\frac{d}{x_{n-3}^{2 j+1}}\right]^{\frac{-1}{2 j+1}}, \\
y_{n+1}=\left[\frac{a}{x_{n}^{2 j+1}}+\frac{b}{y_{n-1}^{2 k+1}}+\frac{c}{x_{n-2}^{2 j+1}}+\frac{d}{y_{n-3}^{2 k+1}}\right]^{\frac{-1}{2 k+1}}, n \in \mathbb{N}_{0},
\end{array}\right.
$$


or equivalently

$$
\left\{\begin{array}{l}
x_{n+1}=\left[\frac{y_{n}^{2 k+1} x_{n-1}^{2 j+1} y_{n-2}^{2 k+1} x_{n-3}^{2 j+1}}{a x_{n-1}^{2 j+1} y_{n-2}^{2 k+1} x_{n-3}^{2 j+1}+b y_{n}^{2 k+1} y_{n-2}^{2 k+2} x_{n-3}^{2 j+1}+c y_{n}^{2 k+1} x_{n-1}^{j+1} x_{n-3}^{2 j+1}+d y_{n}^{2 k+1} x_{n-1}^{2 j+1} y_{n-2}^{2 k+1}}\right]^{\frac{1}{2 j+1}}, \\
y_{n+1}=\left[\frac{x_{n}^{2 j+1} y_{n-1}^{2 k+1} x_{n-2}^{2 j+1} y_{n-3}^{2 k+1}}{a y_{n-1}^{2 k+1} x_{n-2}^{2 j+1} y_{n-3}^{2 k+1}+b x_{n}^{2 j+1} x_{n-2}^{2 j+1} y_{n-3}^{2 k+1}+c x_{n}^{2 j+1} y_{n-1}^{2 k+1} y_{n-3}^{2 k+1}+d x_{n}^{2 j+1} y_{n-1}^{2 k+1} x_{n-2}^{2 j+1}}\right]^{\frac{1}{2 k+1}}, n \in \mathbb{N}_{0} .
\end{array}\right.
$$

Then from (2.2) and (2.3), we obtain that general solution of system (2.19) is

$$
\left\{\begin{aligned}
x_{2 n-1} & =\left[d J_{2 n} x_{-3}^{-(2 j+1)}+\left(c J_{2 n}+d J_{2 n-1}\right) y_{-2}^{-(2 k+1)}+\left(J_{2 n+2}-a J_{2 n+1}\right) x_{-1}^{-(2 j+1)}\right. \\
& \left.+J_{2 n+1} y_{0}^{-(2 k+1)}\right]^{-\frac{1}{2 j+1}}, n \in \mathbb{N}, \\
& =\left[d J_{2 n+1} y_{-3}^{-(2 k+1)}+\left(c J_{2 n+1}+d J_{2 n}\right) x_{-2}^{-(2 j+1)}+\left(J_{2 n+3}-a J_{2 n+2}\right) y_{-1}^{-(2 k+1)}\right. \\
& \left.+J_{2 n+2} x_{0}^{-(2 j+1)}\right]^{-\frac{1}{2 j+1}}, n \in \mathbb{N}_{0},
\end{aligned}\right.
$$

$$
\left\{\begin{aligned}
y_{2 n-1} & =\left[d J_{2 n} y_{-3}^{-(2 k+1)}+\left(c J_{2 n}+d J_{2 n-1}\right) x_{-2}^{-(2 j+1)}+\left(J_{2 n+2}-a J_{2 n+1}\right) y_{-1}^{-(2 k+1)}\right. \\
& \left.+J_{2 n+1} x_{0}^{-(2 j+1)}\right]^{-\frac{1}{2 k+1}}, n \in \mathbb{N}, \\
y_{2 n} & =\left[d J_{2 n+1} x_{-3}^{-(2 j+1)}+\left(c J_{2 n+1}+d J_{2 n}\right) y_{-2}^{-(2 k+1)}+\left(J_{2 n+3}-a J_{2 n+2}\right) x_{-1}^{-(2 j+1)}\right. \\
& \left.+J_{2 n+2} y_{0}^{-(2 k+1)}\right]^{-\frac{1}{2 k+1}}, n \in \mathbb{N}_{0} .
\end{aligned}\right.
$$

If $j=k=0$, then system (2.18) becomes

$$
\left\{\begin{array}{l}
x_{n+1}=\frac{y_{n} x_{n-1} y_{n-2} x_{n-3}}{a x_{n-1} y_{n-2} x_{n-3}+b y_{n} y_{y_{2}-2} x_{n-3}+c y_{n} x_{n-1} x_{n-3}+d y_{n} x_{n-1} y_{n-2}}, \\
y_{n+1}=\frac{x_{n} y_{n-1} x_{n-2} y_{n-3}}{a y_{n-1} x_{n-2} y_{n-3}+b x_{n} x_{n-2} y_{n-3}+c x_{n} y_{n-1} y_{n-3}+d x_{n} y_{n-1} x_{n-2}}, n \in \mathbb{N}_{0} .
\end{array}\right.
$$

The form of the well-defined solutions of (2.22), can be obtained by putting $j=k=0$, in the formulas of the solutions of (2.18). The solutions of the equation, see [15]

$$
x_{n+1}=\frac{x_{n} x_{n-1} x_{n-2} x_{n-3}}{a x_{n-1} x_{n-2} x_{n-3}+b x_{n} x_{n-2} x_{n-3}+c x_{n} x_{n-1} x_{n-3}+d x_{n} x_{n-1} x_{n-2}}, n \in \mathbb{N}_{0},
$$

can be obtained from the solutions of (2.22) by taking $y_{-i}=x_{-i}, i=0,1,2,3$. 


\subsection{Particular cases of system (2.1)}

\subsubsection{The case $d=0$ and $c \neq 0$}

In this case the system (2.1) takes the form:

$$
\left\{\begin{array}{l}
x_{n+1}=f^{-1}\left(a g\left(y_{n}\right)+b f\left(x_{n-1}\right)+c g\left(y_{n-2}\right)\right), \\
y_{n+1}=g^{-1}\left(a f\left(x_{n}\right)+b g\left(y_{n-1}\right)+c f\left(x_{n-2}\right)\right), n \in \mathbb{N}_{0} .
\end{array}\right.
$$

Using the change of variables (2.5), with $n \geq-2$, we get the third order linear system

$$
X_{n+1}=a Y_{n}+b X_{n-1}+c Y_{n-2}, Y_{n+1}=a X_{n}+b Y_{n-1}+c X_{n-2}, n \geq-2 .
$$

Consider the sequence $\left(\widetilde{J}_{n}\right)_{n \geq 0}$ defined by

$$
\widetilde{J}_{n+3}=a \widetilde{J}_{n+2}+b \widetilde{J}_{n+1}+c \widetilde{J}_{n}, \quad n \in \mathbb{N}_{0},
$$

and the special initial values

$$
\widetilde{J_{0}}=0, \widetilde{J_{1}}=1, \widetilde{J_{2}}=a
$$

The sequence $\left(\widetilde{J}_{n}\right)_{n \geq 0}$ is obtained from the sequence $\left(J_{n}\right)_{n \geq 0}$ defined by (1.3):

$$
J_{n+4}=a J_{n+3}+b J_{n+2}+c J_{n+1}+d J_{n}, J_{0}=0, \quad J_{1}=0, \quad J_{2}=1 \text { and } J_{3}=a, n \in \mathbb{N}_{0} .
$$

For $d=0$, we obtain

$$
J_{n+4}=a J_{n+3}+b J_{n+2}+c J_{n+1} .
$$

Putting

$$
\widetilde{J}_{n}=J_{n+1}, n \in \mathbb{N}_{0},
$$

we get the sequence (2.26). Noting that in this case, the corresponding sequences $\left(R_{n}\right)_{n \geq 0},\left(S_{n}\right)_{n \geq 0}$ will be

$$
R_{n+1}=a R_{n}+b R_{n-1}+c R_{n-2}, S_{n+1}=-a S_{n}+b S_{n-1}-c S_{n-2}, n \in \mathbb{N}_{0},
$$

with the initial values $R_{0}, R_{-1}, R_{-2}, S_{0}, S_{-1}, S_{-2}$. The formulas of the solutions of these equations are expressed using the sequence $\left(\widetilde{J}_{n}\right)_{n \geq 0}$, see [2].

The formulas of the solutions of (2.25) and (2.24), can be obtaining from those of solutions of (1.7) and solutions of (2.1) by changing $J_{n}$ by $\widetilde{J}_{n-1}$.

In summary we have the following result.

Corollary 1. Let $\left\{x_{n}, y_{n}\right\}_{n \geq-2}$ be a well-defined solution of system (2.24), then

$$
\begin{aligned}
x_{2 n-1} & =f^{-1}\left[c \widetilde{J}_{2 n-1} g\left(y_{-2}\right)+\left(\widetilde{J}_{2 n+1}-a \widetilde{J}_{2 n}\right) f\left(x_{-1}\right)+\widetilde{J}_{2 n} g\left(y_{0}\right)\right], n \in \mathbb{N}, \\
x_{2 n} & =f^{-1}\left[c \widetilde{J}_{2 n} f\left(x_{-2}\right)+\left(\widetilde{J}_{2 n+2}-a \widetilde{J}_{2 n+1}\right) g\left(y_{-1}\right)+\widetilde{J}_{2 n+1} f\left(x_{0}\right)\right], n \in \mathbb{N}_{0}, \\
y_{2 n-1} & =g^{-1}\left[c \widetilde{J}_{2 n-1} f\left(x_{-2}\right)+\left(\widetilde{J}_{2 n+1}-a \widetilde{J}_{2 n}\right) g\left(y_{-1}\right)+\widetilde{J}_{2 n} f\left(x_{0}\right)\right], n \in \mathbb{N},
\end{aligned}
$$




$$
y_{2 n}=g^{-1}\left[c \widetilde{J}_{2 n} g\left(y_{-2}\right)+\left(\widetilde{J}_{2 n+2}-a \widetilde{J}_{2 n+1}\right) f\left(x_{-1}\right)+\widetilde{J}_{2 n+1} g\left(y_{0}\right)\right], n \in \mathbb{N}_{0} .
$$

Remark 2. If $g \equiv f$ and $y_{-i}=x_{-i}, i=0,1,2$ then, system (2.24) becomes

$$
x_{n+1}=f^{-1}\left[a f\left(x_{n}\right)+b f\left(x_{n-1}\right)+c f\left(x_{n-2}\right)\right]
$$

and by Corollary 1 , the every well defined solution is given by

$$
\left\{\begin{array}{l}
x_{2 n-1}=f^{-1}\left[c \widetilde{J}_{2 n-1} f\left(x_{-2}\right)+\left(\widetilde{J}_{2 n+1}-a \widetilde{J}_{2 n}\right) f\left(x_{-1}\right)+\widetilde{J}_{2 n} f\left(x_{0}\right)\right], n \in \mathbb{N} \\
x_{2 n}=f^{-1}\left[c \widetilde{J}_{2 n} f\left(x_{-2}\right)+\left(\widetilde{J}_{2 n+2}-a \widetilde{J}_{2 n+1}\right) f\left(x_{-1}\right)+\widetilde{J}_{2 n+1} f\left(x_{0}\right)\right], n \in \mathbb{N}_{0}
\end{array}\right.
$$

which can written in a unified form as

$$
x_{n}=f^{-1}\left[c \widetilde{J}_{n} f\left(x_{-2}\right)+\left(\widetilde{J}_{n+2}-a \widetilde{J}_{n+1}\right) f\left(x_{-1}\right)+\widetilde{J}_{n+1} f\left(x_{0}\right)\right], n \in \mathbb{N}_{0} .
$$

Noting again that this equation, was studied by Stevic in [15].

2.2.2. Case $d=0, c \neq 0$ and $a=0$

In this case we get the system

$$
\left\{\begin{array}{l}
x_{n+1}=f^{-1}\left[b f\left(x_{n-1}\right)+c g\left(y_{n-2}\right)\right], \\
y_{n+1}=g^{-1}\left[b g\left(y_{n-1}\right)+c f\left(x_{n-2}\right)\right], n \in \mathbb{N}_{0} .
\end{array}\right.
$$

Here, $\left(\widetilde{J}_{n}\right)_{n \geq 0}$ will be the sequence defined by

$$
\mathcal{P}_{n+3}=b \mathcal{P}_{n+1}+c \mathcal{P}_{n}, \quad n \in \mathbb{N}_{0},
$$

and the special initial values

$$
\mathcal{P}_{0}=0, \quad \mathcal{P}_{1}=1 \text { and } \mathcal{P}_{2}=0,
$$

so, the solutions are expressed in terms of $(\mathscr{P})_{n \geq 0}$ and are given by

$$
\begin{aligned}
x_{2 n-1} & =f^{-1}\left[c \mathcal{P}_{2 n-1} g\left(y_{-2}\right)+\mathcal{P}_{2 n+1} f\left(x_{-1}\right)+\mathcal{P}_{2 n} g\left(y_{0}\right)\right], n \in \mathbb{N}, \\
x_{2 n} & =f^{-1}\left[c \mathcal{P}_{2 n} f\left(x_{-2}\right)+\mathcal{P}_{2 n+2} g\left(y_{-1}\right)+\mathcal{P}_{2 n+1} f\left(x_{0}\right)\right], n \in \mathbb{N}_{0}, \\
y_{2 n-1} & =g^{-1}\left[c \mathcal{P}_{2 n-1} f\left(x_{-2}\right)+\mathcal{P}_{2 n+1} g\left(y_{-1}\right)+\mathcal{P}_{2 n} f\left(x_{0}\right)\right], n \in \mathbb{N}, \\
y_{2 n} & =g^{-1}\left[c \mathcal{P}_{2 n} g\left(y_{-2}\right)+\mathcal{P}_{2 n+2} f\left(x_{-1}\right)+\mathcal{P}_{2 n+1} g\left(y_{0}\right)\right], n \in \mathbb{N}_{0},
\end{aligned}
$$

for the system (2.30) and by

$$
x_{n}=f^{-1}\left[c \mathcal{P}_{n} f\left(x_{-2}\right)+\mathcal{P}_{n+2} f\left(x_{-1}\right)+\mathcal{P}_{n+1} f\left(x_{0}\right)\right], n \in \mathbb{N}_{0},
$$

for its one dimensional version, that is the equation

$$
x_{n+1}=f^{-1}\left(b f\left(x_{n-1}\right)+c f\left(x_{n-2}\right)\right) .
$$

If $b \neq 0,(\mathcal{P})_{n \geq 0}$ will be a generalized Padovan sequence and if $b=c=1$, then $\left(\mathscr{P}_{n}\right)_{n \geq 0}$ will be the famous Padovan sequence.

Noting that system (2.30) generalize for example the works of [5] and [21]. 
2.2.3. Case $c=d=0$ and $b \neq 0$

In this case, we get the system

$$
\left\{\begin{array}{l}
x_{n+1}=f^{-1}\left[a g\left(y_{n}\right)+b f\left(x_{n-1}\right)\right], \\
y_{n+1}=g^{-1}\left[a f\left(x_{n}\right)+b g\left(y_{n-1}\right)\right], n \in \mathbb{N}_{0} .
\end{array}\right.
$$

By the same philosophy, we obtain the sequence $\left(\widetilde{F}_{n}\right)_{n \geq 0}=\left(\widetilde{J}_{n+1}\right)_{n \geq 0}$, defined by

$$
\widetilde{F}_{n+2}=a \widetilde{F}_{n+1}+b \widetilde{F}_{n}, \widetilde{F}_{0}=1, \widetilde{F}_{1}=a, n \in \mathbb{N}_{0},
$$

and the solutions of (2.33) and its one dimensional version, are obtained from the solutions of (2.24) and (2.27), by writing $\widetilde{F}_{n-1}$ instead of $\widetilde{J}_{n}$. If $a \neq 0,\left(\widetilde{F}_{n}\right)_{n \geq 0}$ is a generalized Fibonacci sequence and if $a=b=1,\left(\widetilde{F}_{n}\right)_{n \geq 0}$ will be the famous Fibonacci sequence.

System (2.33) and its one dimensional versions, generalize for example the works of [17], [18].

\subsubsection{Case $b=c=d=0$ and $a \neq 0$}

In this case, we get the system

$$
\left.x_{n+1}=f^{-1}\left(\operatorname{ag}\left(y_{n}\right)\right)\right), y_{n+1}=g^{-1}\left(a f\left(x_{n}\right)\right), n \in \mathbb{N}_{0} .
$$

Using the fact that $f, g$ are one to one functions, and the change of variables

$$
X_{n}=f\left(x_{n}\right), Y_{n}=g\left(y_{n}\right), n \geq 0
$$

the system (2.34), will be

$$
X_{n+1}=a Y_{n}, Y_{n+1}=a X_{n}, n \in \mathbb{N}_{0} .
$$

So,

$$
X_{2 n}=a^{2 n} X_{0}, Y_{2 n}=a^{2 n} Y_{0}, X_{2 n+1}=a^{2 n+1} Y_{0}, Y_{2 n+1}=a^{2 n+1} X_{0}, n \in \mathbb{N}_{0} .
$$

Hence

and

$$
x_{2 n}=f^{-1}\left(a^{2 n} f\left(x_{0}\right)\right), y_{2 n}=g^{-1}\left(a^{2 n} g\left(y_{0}\right)\right), n \in \mathbb{N}_{0}
$$

$$
x_{2 n+1}=f^{-1}\left(a^{2 n+1} g\left(y_{0}\right)\right), y_{2 n+1}=g^{-1}\left(a^{2 n+1} f\left(x_{0}\right)\right), n \in \mathbb{N}_{0} .
$$

3. SOLUTIONS OF A SECOND GENERAL SYSTEM OF DIFFERENCE EQUATIONS

In this part, we are interested in the following system of difference equations given by

$$
\left\{\begin{array}{l}
x_{n+1}=f^{-1}\left(a+\frac{b}{g\left(y_{n}\right)}+\frac{c}{g\left(y_{n}\right) f\left(x_{n-1}\right)}+\frac{d}{g\left(y_{n}\right) f\left(x_{n-1}\right) g\left(y_{n-2}\right)}\right), \\
y_{n+1}=g^{-1}\left(a+\frac{b}{f\left(x_{n}\right)}+\frac{c}{f\left(x_{n}\right) g\left(y_{n-1}\right)}+\frac{d}{f\left(x_{n}\right) g\left(y_{n-1}\right) f\left(x_{n-2}\right)}\right),
\end{array}\right.
$$


where $n \in \mathbb{N}_{0}, f, g: D \longrightarrow \mathbb{R}$ are continuous functions, with $D=D_{f}=D_{g}$, that is $f$ and $g$ have the same domain, in addition we assume that $f, g$ are " $1-1$ " on $D \subseteq \mathbb{R}$, the initial values $x_{-i}, y_{-i}, i=0,1,2$, are arbitrary real numbers in $D$ and the parameters $a, b, c$ and $d$ are arbitrary real numbers.

Definition 2. A solution $\left\{x_{n}, y_{n}\right\}_{n>-2}$ of system (3.1) is said to be well-defined if for all $n \in \mathbb{N}_{0}$, we have

and

$$
a+\frac{b}{g\left(y_{n}\right)}+\frac{c}{g\left(y_{n}\right) f\left(x_{n-1}\right)}+\frac{d}{g\left(y_{n}\right) f\left(x_{n-1}\right) g\left(y_{n-2}\right)} \in D_{f^{-1}},
$$

$$
a+\frac{b}{f\left(x_{n}\right)}+\frac{c}{f\left(x_{n}\right) g\left(y_{n-1}\right)}+\frac{d}{f\left(x_{n}\right) g\left(y_{n-1}\right) f\left(x_{n-2}\right)} \in D_{g^{-1}} .
$$

We solve in closed form (3.1) and we investigated particular cases of it. The philosophy is the same as in the previous section (2), so we will brief in presenting our formulas of the solutions.

\subsection{Explicit formulas of solutions of system (3.1) with $d \neq 0$}

The following result is devoted to the formulas of well-defined solutions of (3.1).

Theorem 2. Let $\left\{x_{n}, y_{n}\right\}_{n>-2}$ be a well-defined solution of system (3.1). Then, for all $n \in \mathbb{N}_{0}$ we have

$$
\begin{aligned}
x_{2 n+1} & =f^{-1}\left[\left(d J_{2 n+2}+\left(c J_{2 n+2}+d J_{2 n+1}\right) g\left(y_{-2}\right)+\left(J_{2 n+4}-a J_{2 n+3}\right) f\left(x_{-1}\right) g\left(y_{-2}\right)\right.\right. \\
& \left.+J_{2 n+3} g\left(y_{0}\right) f\left(x_{-1}\right) g\left(y_{-2}\right)\right)\left(d J_{2 n+1}+\left(c J_{2 n+1}+d J_{2 n}\right) g\left(y_{-2}\right)\right. \\
& \left.\left.+\left(J_{2 n+3}-a J_{2 n+2}\right) f\left(x_{-1}\right) g\left(y_{-2}\right)+J_{2 n+2} g\left(y_{0}\right) f\left(x_{-1}\right) g\left(y_{-2}\right)\right)^{-1}\right], \\
x_{2 n+2} & =f^{-1}\left[\left(d J_{2 n+3}+\left(c J_{2 n+3}+d J_{2 n+2}\right) f\left(x_{-2}\right)+\left(J_{2 n+5}-a J_{2 n+4}\right) g\left(y_{-1}\right) f\left(x_{-2}\right)\right.\right. \\
& \left.+J_{2 n+4} f\left(x_{0}\right) g\left(y_{-1}\right) f\left(x_{-2}\right)\right)\left(d J_{2 n+2}+\left(c J_{2 n+2}+d J_{2 n+1}\right) f\left(x_{-2}\right)\right. \\
& \left.\left.+\left(J_{2 n+4}-a J_{2 n+3}\right) g\left(y_{-1}\right) f\left(x_{-2}\right)+J_{2 n+3} f\left(x_{0}\right) g\left(y_{-1}\right) f\left(x_{-2}\right)\right)^{-1}\right], \\
y_{2 n+1} & =g^{-1}\left[\left(d J_{2 n+2}+\left(c J_{2 n+2}+d J_{2 n+1}\right) f\left(x_{-2}\right)+\left(J_{2 n+4}-a J_{2 n+3}\right) g\left(y_{-1}\right) f\left(x_{-2}\right)\right.\right. \\
& \left.+J_{2 n+3} f\left(x_{0}\right) g\left(y_{-1}\right) f\left(x_{-2}\right)\right)\left(d J_{2 n+1}+\left(c J_{2 n+1}+d J_{2 n}\right) f\left(x_{-2}\right)\right. \\
& \left.\left.+\left(J_{2 n+3}-a J_{2 n+2}\right) g\left(y_{-1}\right) f\left(x_{-2}\right)+J_{2 n+2} f\left(x_{0}\right) g\left(y_{-1}\right) f\left(x_{-2}\right)\right)^{-1}\right], \\
y_{2 n+2} & =g^{-1}\left[\left(d J_{2 n+3}+\left(c J_{2 n+3}+d J_{2 n+2}\right) g\left(y_{-2}\right)+\left(J_{2 n+5}-a J_{2 n+4}\right) f\left(x_{-1}\right) g\left(y_{-2}\right)\right.\right. \\
& \left.+J_{2 n+4} g\left(y_{0}\right) f\left(x_{-1}\right) g\left(y_{-2}\right)\right)\left(d J_{2 n+2}+\left(c J_{2 n+2}+d J_{2 n+1}\right) g\left(y_{-2}\right)\right. \\
& \left.\left.+\left(J_{2 n+4}-a J_{2 n+3}\right) f\left(x_{-1}\right) g\left(y_{-2}\right)+J_{2 n+3} g\left(y_{0}\right) f\left(x_{-1}\right) g\left(y_{-2}\right)\right)^{-1}\right] .
\end{aligned}
$$

Proof. Using the fact that the functions $f, g$ are one to one and using the change of variables (2.5), with $n \geq-2$, the system (3.1) becomes

$$
\left\{\begin{array}{l}
X_{n+1}=a+\frac{b}{Y_{n}}+\frac{c}{Y_{n} X_{n-1}}+\frac{d}{Y_{n} X_{n-1} Y_{n-2}}, \\
Y_{n+1}=a+\frac{b}{X_{n}}+\frac{c}{X_{n} Y_{n-1}}+\frac{d}{X_{n} Y_{n-1} X_{n-2}}, n \in \mathbb{N}_{0},
\end{array}\right.
$$


or equivalently,

$$
\left\{\begin{array}{l}
X_{n+1}=\frac{a Y_{n} X_{n-1} Y_{n-2}+b X_{n-1} Y_{n-2}+c Y_{n-2}+d}{Y_{n} X_{n-1} Y_{n-2}}, \\
Y_{n+1}=\frac{a X_{n} Y_{n-1} X_{n-2}+b Y_{n-1} X_{n-2}+c X_{n-2}+d}{X_{n} Y_{n-1} X_{n-2}}, n \in \mathbb{N}_{0} .
\end{array}\right.
$$

This system was solved in [2], and for $n \in \mathbb{N}_{0}$, the solutions of (3.3) takes the form

$X_{2 n+1}=\frac{d J_{2 n+2}+\left(c J_{2 n+2}+d J_{2 n+1}\right) Y_{-2}+\left(J_{2 n+4}-a J_{2 n+3}\right) X_{-1} Y_{-2}+J_{2 n+3} Y_{0} X_{-1} Y_{-2}}{d J_{2 n+1}+\left(c J_{2 n+1}+d J_{2 n}\right) Y_{-2}+\left(J_{2 n+3}-a J_{2 n+2}\right) X_{-1} Y_{-2}+J_{2 n+2} Y_{0} X_{-1} Y_{-2}}$,

$X_{2 n+2}=\frac{d J_{2 n+3}+\left(c J_{2 n+3}+d J_{2 n+2}\right) X_{-2}+\left(J_{2 n+5}-a J_{2 n+4}\right) Y_{-1} X_{-2}+J_{2 n+4} X_{0} Y_{-1} X_{-2}}{d J_{2 n+2}+\left(c J_{2 n+2}+d J_{2 n+1}\right) X_{-2}+\left(J_{2 n+4}-a J_{2 n+3}\right) Y_{-1} X_{-2}+J_{2 n+3} X_{0} Y_{-1} X_{-2}}$,

$Y_{2 n+1}=\frac{d J_{2 n+2}+\left(c J_{2 n+2}+d J_{2 n+1}\right) X_{-2}+\left(J_{2 n+4}-a J_{2 n+3}\right) Y_{-1} X_{-2}+J_{2 n+3} X_{0} Y_{-1} X_{-2}}{d J_{2 n+1}+\left(c J_{2 n+1}+d J_{2 n}\right) X_{-2}+\left(J_{2 n+3}-a J_{2 n+2}\right) Y_{-1} X_{-2}+J_{2 n+2} X_{0} Y_{-1} X_{-2}}$,

$Y_{2 n+2}=\frac{d J_{2 n+3}+\left(c J_{2 n+3}+d J_{2 n+2}\right) Y_{-2}+\left(J_{2 n+5}-a J_{2 n+4}\right) X_{-1} Y_{-2}+J_{2 n+4} Y_{0} X_{-1} Y_{-2}}{d J_{2 n+2}+\left(c J_{2 n+2}+d J_{2 n+1}\right) Y_{-2}+\left(J_{2 n+4}-a J_{2 n+3}\right) X_{-1} Y_{-2}+J_{2 n+3} Y_{0} X_{-1} Y_{-2}}$,

where $\left(J_{n}\right)_{n \in \mathbb{N}_{0}}$ is the sequence defined by (1.3).

Using (2.5), (3.4), (3.5), (3.6) and (3.7), we get that for $n \in \mathbb{N}_{0}$, every well-defined solution of system (3.1) has the following representation

$$
\begin{aligned}
x_{2 n+1} & =f^{-1}\left[\left(d J_{2 n+2}+\left(c J_{2 n+2}+d J_{2 n+1}\right) g\left(y_{-2}\right)+\left(J_{2 n+4}-a J_{2 n+3}\right) f\left(x_{-1}\right) g\left(y_{-2}\right)\right.\right. \\
& \left.+J_{2 n+3} g\left(y_{0}\right) f\left(x_{-1}\right) g\left(y_{-2}\right)\right)\left(d J_{2 n+1}+\left(c J_{2 n+1}+d J_{2 n}\right) g\left(y_{-2}\right)\right. \\
& \left.\left.+\left(J_{2 n+3}-a J_{2 n+2}\right) f\left(x_{-1}\right) g\left(y_{-2}\right)+J_{2 n+2} g\left(y_{0}\right) f\left(x_{-1}\right) g\left(y_{-2}\right)\right)^{-1}\right], \\
x_{2 n+2} & =f^{-1}\left[\left(d J_{2 n+3}+\left(c J_{2 n+3}+d J_{2 n+2}\right) f\left(x_{-2}\right)+\left(J_{2 n+5}-a J_{2 n+4}\right) g\left(y_{-1}\right) f\left(x_{-2}\right)\right.\right. \\
& \left.+J_{2 n+4} f\left(x_{0}\right) g\left(y_{-1}\right) f\left(x_{-2}\right)\right)\left(d J_{2 n+2}+\left(c J_{2 n+2}+d J_{2 n+1}\right) f\left(x_{-2}\right)\right. \\
& \left.\left.+\left(J_{2 n+4}-a J_{2 n+3}\right) g\left(y_{-1}\right) f\left(x_{-2}\right)+J_{2 n+3} f\left(x_{0}\right) g\left(y_{-1}\right) f\left(x_{-2}\right)\right)^{-1}\right], \\
y_{2 n+1} & =g^{-1}\left[\left(d J_{2 n+2}+\left(c J_{2 n+2}+d J_{2 n+1}\right) f\left(x_{-2}\right)+\left(J_{2 n+4}-a J_{2 n+3}\right) g\left(y_{-1}\right) f\left(x_{-2}\right)\right.\right. \\
& \left.+J_{2 n+3} f\left(x_{0}\right) g\left(y_{-1}\right) f\left(x_{-2}\right)\right)\left(d J_{2 n+1}+\left(c J_{2 n+1}+d J_{2 n}\right) f\left(x_{-2}\right)\right. \\
& \left.\left.+\left(J_{2 n+3}-a J_{2 n+2}\right) g\left(y_{-1}\right) f\left(x_{-2}\right)+J_{2 n+2} f\left(x_{0}\right) g\left(y_{-1}\right) f\left(x_{-2}\right)\right)^{-1}\right], \\
y_{2 n+2} & =g^{-1}\left[\left(d J_{2 n+3}+\left(c J_{2 n+3}+d J_{2 n+2}\right) g\left(y_{-2}\right)+\left(J_{2 n+5}-a J_{2 n+4}\right) f\left(x_{-1}\right) g\left(y_{-2}\right)\right.\right. \\
& \left.+J_{2 n+4} g\left(y_{0}\right) f\left(x_{-1}\right) g\left(y_{-2}\right)\right)\left(d J_{2 n+2}+\left(c J_{2 n+2}+d J_{2 n+1}\right) g\left(y_{-2}\right)\right. \\
& \left.\left.+\left(J_{2 n+4}-a J_{2 n+3}\right) f\left(x_{-1}\right) g\left(y_{-2}\right)+J_{2 n+3} g\left(y_{0}\right) f\left(x_{-1}\right) g\left(y_{-2}\right)\right)^{-1}\right] .
\end{aligned}
$$


Remark 3. (1) In [2], to solve the system (3.3), the authors used the change of variables

$$
X_{n}=\frac{u_{n}}{v_{n-1}}, Y_{n}=\frac{v_{n}}{u_{n-1}}, n \geq-2,
$$

to obtain the fourth linear system (1.7).

(2) When $g \equiv f$ and $y_{-i}=x_{-i}, i=\overline{0,2}$ then system (3.1) becomes the equation

$$
x_{n+1}=f^{-1}\left[a+\frac{b}{f\left(x_{n}\right)}+\frac{c}{f\left(x_{n}\right) f\left(x_{n-1}\right)}+\frac{d}{f\left(x_{n}\right) f\left(x_{n-1}\right) f\left(x_{n-2}\right)}\right], n \in \mathbb{N}_{0} \text {, }
$$

and the form of every well-defined solution of (3.8) is given by

$$
\begin{aligned}
x_{2 n+1} & =f^{-1}\left[\left(d J_{2 n+2}+\left(c J_{2 n+2}+d J_{2 n+1}\right) f\left(x_{-2}\right)+\left(J_{2 n+4}-a J_{2 n+3}\right) f\left(x_{-1}\right) f\left(x_{-2}\right)\right.\right. \\
& \left.+J_{2 n+3} f\left(x_{0}\right) f\left(x_{-1}\right) f\left(x_{-2}\right)\right)\left(d J_{2 n+1}+\left(c J_{2 n+1}+d J_{2 n}\right) f\left(x_{-2}\right)\right. \\
& \left.\left.+\left(J_{2 n+3}-a J_{2 n+2}\right) f\left(x_{-1}\right) f\left(x_{-2}\right)+J_{2 n+2} f\left(x_{0}\right) f\left(x_{-1}\right) f\left(x_{-2}\right)\right)^{-1}\right], \\
x_{2 n+2} & =f^{-1}\left[\left(d J_{2 n+3}+\left(c J_{2 n+3}+d J_{2 n+2}\right) f\left(x_{-2}\right)+\left(J_{2 n+5}-a J_{2 n+4}\right) f\left(x_{-1}\right) f\left(x_{-2}\right)\right.\right. \\
& \left.+J_{2 n+4} f\left(x_{0}\right) f\left(x_{-1}\right) f\left(x_{-2}\right)\right)\left(d J_{2 n+2}+\left(c J_{2 n+2}+d J_{2 n+1}\right) f\left(x_{-2}\right)\right. \\
& \left.\left.+\left(J_{2 n+4}-a J_{2 n+3}\right) f\left(x_{-1}\right) f\left(x_{-2}\right)+J_{2 n+3} f\left(x_{0}\right) f\left(x_{-1}\right) f\left(x_{-2}\right)\right)^{-1}\right],
\end{aligned}
$$

which can be represented in the unified form

$$
\begin{aligned}
x_{n+1} & =f^{-1}\left[\left(d J_{n+2}+\left(c J_{n+2}+d J_{n+1}\right) f\left(x_{-2}\right)+\left(J_{n+4}-a J_{n+3}\right) f\left(x_{-1}\right) f\left(x_{-2}\right)\right.\right. \\
& \left.+J_{n+3} f\left(x_{0}\right) f\left(x_{-1}\right) f\left(x_{-2}\right)\right)\left(d J_{n+1}+\left(c J_{n+1}+d J_{n}\right) f\left(x_{-2}\right)\right. \\
& \left.\left.+\left(J_{n+3}-a J_{n+2}\right) f\left(x_{-1}\right) f\left(x_{-2}\right)+J_{n+2} f\left(x_{0}\right) f\left(x_{-1}\right) f\left(x_{-2}\right)\right)^{-1}\right] .
\end{aligned}
$$

Now we give some applications of Theorem 2.

Example 3. Let

$$
f(t)=t^{2 j+1}, g(t)=t^{2 k+1}, j, k \in \mathbb{N}_{0} .
$$

We have the functions $f$ and $g$ are one to one continuous functions on $\mathbb{R}=D_{f}=D_{g}$. In this case, system (3.1) becomes

$$
\left\{\begin{array}{l}
x_{n+1}=\left[a+\frac{b}{y_{n}^{2 k+1}}+\frac{c}{y_{n}^{2 k+1} x_{n-1}^{2 j+1}}+\frac{d}{y_{n}^{2 k+1} x_{n-1}^{2 j+1} y_{n-2}^{2 k+1}}\right]^{\frac{1}{2 j+1}}, \\
y_{n+1}=\left[a+\frac{b}{x_{n}^{2 j+1}}+\frac{c}{x_{n}^{2 j+1} y_{n-1}^{2 k+1}}+\frac{d}{x_{n}^{2 j+1} y_{n-1}^{2 k+1} x_{n-2}^{2 j+1}}\right]^{\frac{1}{2 k+1}}, n \in \mathbb{N}_{0} .
\end{array}\right.
$$

Then by Theorem 2, we obtain that the solutions of system (3.9) have the following form

$$
\begin{aligned}
& x_{2 n+1}=\left[\frac{d J_{2 n+2}+\left(c J_{2 n+2}+d J_{2 n+1}\right) y_{-2}^{2 k+1}+\left(J_{2 n+4}-a J_{2 n+3}\right) x_{-1}^{2 j+1} y_{-2}^{2 k+1}+J_{2 n+3} y_{0}^{2 k+1} x_{-1}^{2 j+1} y_{-2}^{2 k+1}}{d J_{2 n+1}+\left(c J_{2 n+1}+d J_{2 n}\right) y_{-2}^{2 k+1}+\left(J_{2 n+3}-a J_{2 n+2}\right) x_{-1}^{2 j+1} y_{-2}^{2 k+1}+J_{2 n+2}^{2} y_{0}^{2 k+1} x_{-1}^{2 j+1} y_{-2}^{2 k+1}}\right]^{\frac{1}{2 j+1}}, \\
& x_{2 n+2}=\left[\frac{d J_{2 n+3}+\left(c J_{2 n+3}+d J_{2 n+2}\right) x_{-2}^{2 j+1}+\left(J_{2 n+5}-a J_{2 n+4}\right) y_{-1}^{2 k+1} x_{-2}^{2 j+1}+J_{2 n+4} x_{0}^{2 j+1} y_{-1}^{2 k+1} x_{-2}^{2 j+1}}{d J_{2 n+2}+\left(c J_{2 n+2}+d J_{2 n+1}\right) x_{-2}^{2 j+1}+\left(J_{2 n+4}-a J_{2 n+3}\right)_{-1}^{2 k+1} x_{-2}^{2 j+1}+J_{2 n+3} x_{0}^{j+1} y_{-1}^{2 k+1} x_{-2}^{2 j+1}}\right]^{\frac{1}{2 j+1}}, \\
& y_{2 n+1}=\left[\frac{d J_{2 n+2}+\left(c J_{2 n+2}+d J_{2 n+1}\right) x_{-2}^{2 j+1}+\left(J_{2 n+4}-a J_{2 n+3}\right) y_{-1}^{2 k+1} x_{-2}^{2 j+1}+J_{2 n+3} x_{0}^{2 j+1} y_{-1}^{2 k+1} x_{-2}^{2 j+1}}{d J_{2 n+1}+\left(c J_{2 n+1}+d J_{2 n}\right) x_{-2}^{2 j+1}+\left(J_{2 n+3}-a J_{2 n+2}\right) y_{-1}^{2 k+1} x_{-2}^{2 j+1}+J_{2 n+2}^{2 j+1} x_{0}^{2 k+1} y_{-1}^{2 k+1} x_{-2}^{2 j+1}}\right]^{\frac{1}{2 k+1}},
\end{aligned}
$$




$$
y_{2 n+2}=\left[\frac{d J_{2 n+3}+\left(c J_{2 n+3}+d J_{2 n+2}\right) y_{-2}^{2 k+1}+\left(J_{2 n+5}-a J_{2 n+4}\right) x_{-1}^{2 j+1} y_{-2}^{2 k+1}+J_{2 n+4} y_{0}^{2 k+1} x_{-1}^{2 j+1} y_{-2}^{2 k+1}}{d J_{2 n+2}+\left(c J_{2 n+2}+d J_{2 n+1}\right) y_{-2}^{2 k+1}+\left(J_{2 n+4}-a J_{2 n+3}\right) x_{-1}^{2 j+1} y_{-2}^{2 k+1}+J_{2 n+3} y_{0}^{2 k+1} x_{-1}^{2 j+1} y_{-2}^{2 k+1}}\right]^{\frac{1}{2 k+1}} .
$$

Example 4. Let

$$
f(t)=\frac{1}{t^{2 j+1}}, g(t)=\frac{1}{t^{2 k+1}}, j, k \in \mathbb{N}_{0} .
$$

We have the functions $f$ and $g$ are one to one continuous functions on $\mathbb{R}-\{0\}=$ $D_{f}=D_{g}$. Then, system (3.1) becomes

$$
\left\{\begin{array}{l}
x_{n+1}=\left[\frac{1}{a+b y_{n}^{2 k+1}+c y_{n}^{2 k+1} x_{n-1}^{2 j+1}+d y_{n}^{2 k+1} x_{n-1}^{2 j+1} y_{n-2}^{2 k+1}}\right]^{\frac{1}{2 j+1}}, \\
y_{n+1}=\left[\frac{1}{a+b x_{n}^{2 j+1}+c x_{n}^{2 j+1} y_{n-1}^{2 k+1}+d x_{n}^{2 j+1} y_{n-1}^{2 k+1} x_{n-2}^{2 j+1}}\right]^{\frac{1}{2 k+1}}, n \in \mathbb{N}_{0} .
\end{array}\right.
$$

Then by Theorem 2, the solutions of system (3.14) have the following representation

$$
\begin{aligned}
x_{2 n+1}= & {\left[\frac{d J_{2 n+1} y_{0}^{2 k+1} x_{-1}^{2 j+1} y_{-2}^{2 k+1}+\left(c J_{2 n+1}+d J_{2 n}\right) y_{0}^{2 k+1} x_{-1}^{2 j+1}+\left(J_{2 n+3}-a J_{2 n+2}\right) y_{0}^{2 k+1}+J_{2 n+2}}{d J_{2 n+2} y_{0}^{2 k+1} x_{-1}^{2 j+1} y_{-2}^{2 k+1}+\left(c J_{2 n+2}+d J_{2 n+1}\right) y_{0}^{2 k+1} x_{-1}^{2 j+1}+\left(J_{2 n+4}-a J_{2 n+3}\right) y_{0}^{2 k+1}+J_{2 n+3}}\right]^{\frac{1}{2 j+1}}, } \\
x_{2 n+2}= & {\left[\frac{d J_{2 n+2} x_{0}^{2 j+1} y_{-1}^{2 k+1} x_{-2}^{2 j+1}+\left(c J_{2 n+2}+d J_{2 n+1}\right) x_{0}^{2 j+1} y_{-1}^{2 k+1}+\left(J_{2 n+4}-a J_{2 n+3}\right) x_{0}^{2 j+1}+J_{2 n+3}}{d J_{2 n+3} x_{0}^{2 j+1} y_{-1}^{2 k+1} x_{-2}^{2 j+1}+\left(c J_{2 n+3}+d J_{2 n+2}\right) x_{0}^{2 j+1} y_{-1}^{2 k+1}+\left(J_{2 n+5}-a J_{2 n+4}\right) x_{0}^{2 j+1}+J_{2 n+4}}\right]^{\frac{1}{2 j+1}}, } \\
y_{2 n+1}= & {\left[\frac{d J_{2 n+1} x_{0}^{2 j+1} y_{-1}^{2 k+1} x_{-2}^{2 j+1}+\left(c J_{2 n+1}+d J_{2 n}\right) x_{0}^{2 j+1} y_{-1}^{2 k+1}+\left(J_{2 n+3}-a J_{2 n+2}\right) x_{0}^{2 j+1}+J_{2 n+2}}{d J_{2 n+2} x_{0}^{2 j+1} y_{-1}^{2 k+1} x_{-2}^{2 j+1}+\left(c J_{2 n+2}+d J_{2 n+1}\right) x_{0}^{2 j+1} y_{-1}^{2 k+1}+\left(J_{2 n+4}-a J_{2 n+3}\right) x_{0}^{2 j+1}+J_{2 n+3}}\right]^{\frac{1}{2 k+1}}, } \\
y_{2 n+2}= & {\left[\frac{d J_{2 n+2} y_{0}^{2 k+1} x_{-1}^{2 j+1} y_{-2}^{2 k+1}+\left(c J_{2 n+2}+d J_{2 n+1}\right) y_{0}^{2 k+1} x_{-1}^{2 j+1}+\left(J_{2 n+4}-a J_{2 n+3}\right) y_{0}^{2 k+1}+J_{2 n+3}}{d J_{2 n+3} y_{0}^{2 k+1} x_{-1}^{2 j+1} y_{-2}^{2 k+1}+\left(c J_{2 n+3}+d J_{2 n+2}\right) y_{0}^{2 k+1} x_{-1}^{2 j+1}+\left(J_{2 n+5}-a J_{2 n+4}\right) y_{0}^{2 k+1}+J_{2 n+4}}\right]^{\frac{1}{2 k+1}} . }
\end{aligned}
$$

3.1.1. The system $x_{n+1}=\frac{1}{a+b y_{n}+c y_{n} x_{n-1}+d y_{n} x_{n-1} y_{n-2}}$,

$$
y_{n+1}=\frac{1}{a+b x_{n}+c x_{n} y_{n-1}+d x_{n} y_{n-1} x_{n-2}}
$$

Here we will focus our study on the system of difference equations

$$
\left\{\begin{array}{l}
x_{n+1}=\frac{1}{a+b y_{n}+c y_{n} x_{n-1}+d y_{n} x_{n-1} y_{n-2}}, \\
y_{n+1}=\frac{1}{a+b x_{n}+c x_{n} y_{n-1}+d x_{n} y_{n-1} x_{n-2}},
\end{array} \quad n \in \mathbb{N}_{0}\right.
$$

which is a particular case of system (3.14) with $j=k=0$. Noting that system (3.19), generalize the studies in [12], [11], [13]. Then, putting $j=k=0$ in the formulas of well-defined solutions of system (3.14), we obtain the following result.

Corollary 2. Let $\left\{x_{n}, y,\right\}_{n \geq-2}$ be a well-defined solution of (3.19), then for $n \in \mathbb{N}_{0}$, we have

$$
x_{2 n+1}=\frac{d J_{2 n+1} y_{0} x_{-1} y_{-2}+\left(c J_{2 n+1}+d J_{2 n}\right) y_{0} x_{-1}+\left(J_{2 n+3}-a J_{2 n+2}\right) y_{0}+J_{2 n+2}}{d J_{2 n+2} y_{0} x_{-1} y_{-2}+\left(c J_{2 n+2}+d J_{2 n+1}\right) y_{0} x_{-1}+\left(J_{2 n+4}-a J_{2 n+3}\right) y_{0}+J_{2 n+3}},
$$




$$
\begin{aligned}
& x_{2 n+2}=\frac{d J_{2 n+2} x_{0} y_{-1} x_{-2}+\left(c J_{2 n+2}+d J_{2 n+1}\right) x_{0} y_{-1}+\left(J_{2 n+4}-a J_{2 n+3}\right) x_{0}+J_{2 n+3}}{d J_{2 n+3} x_{0} y_{-1} x_{-2}+\left(c J_{2 n+3}+d J_{2 n+2}\right) x_{0} y_{-1}+\left(J_{2 n+5}-a J_{2 n+4}\right) x_{0}+J_{2 n+4}}, \\
& y_{2 n+1}=\frac{d J_{2 n+1} x_{0} y_{-1} x_{-2}+\left(c J_{2 n+1}+d J_{2 n}\right) x_{0} y_{-1}+\left(J_{2 n+3}-a J_{2 n+2}\right) x_{0}+J_{2 n+2}}{d J_{2 n+2} x_{0} y_{-1} x_{-2}+\left(c J_{2 n+2}+d J_{2 n+1}\right) x_{0} y_{-1}+\left(J_{2 n+4}-a J_{2 n+3}\right) x_{0}+J_{2 n+3}}, \\
& y_{2 n+2}=\frac{d J_{2 n+2} y_{0} x_{-1} y_{-2}+\left(c J_{2 n+2}+d J_{2 n+1}\right) y_{0} x_{-1}+\left(J_{2 n+4}-a J_{2 n+3}\right) y_{0}+J_{2 n+3}}{d J_{2 n+3} y_{0} x_{-1} y_{-2}+\left(c J_{2 n+3}+d J_{2 n+2}\right) y_{0} x_{-1}+\left(J_{2 n+5}-a J_{2 n+4}\right) y_{0}+J_{2 n+4}} .
\end{aligned}
$$

Moreover, if we choose $a=b=c=d=1$, the sequence $\left(J_{n}\right)_{n=0}^{+\infty}$ will be nothing other than the famous Tetranacci sequence defined for $n \in \mathbb{N}_{0}$ by

$$
T_{n+4}=T_{n+3}+T_{n+2}+T_{n+1}+T_{n}, T_{0}=T_{1}=0, \quad T_{2}=T_{3}=1,
$$

and for this choice of the parameters, the solutions takes the form

$$
\begin{aligned}
x_{2 n+1}= & \frac{T_{2 n+1} y_{0} x_{-1} y_{-2}+\left(T_{2 n+1}+T_{2 n}\right) y_{0} x_{-1}+\left(T_{2 n+3}-T_{2 n+2}\right) y_{0}+T_{2 n+2}}{T_{2 n+2} y_{0} x_{-1} y_{-2}+\left(T_{2 n+2}+T_{2 n+1}\right) y_{0} x_{-1}+\left(T_{2 n+4}-T_{2 n+3}\right) y_{0}+T_{2 n+3}}, \\
x_{2 n+2}= & \frac{T_{2 n+2} x_{0} y_{-1} x_{-2}+\left(T_{2 n+2}+T_{2 n+1}\right) x_{0} y_{-1}+\left(T_{2 n+4}-T_{2 n+3}\right) x_{0}+T_{2 n+3}}{T_{2 n+3} x_{0} y_{-1} x_{-2}+\left(T_{2 n+3}+T_{2 n+2}\right) x_{0} y_{-1}+\left(T_{2 n+5}-T_{2 n+4}\right) x_{0}+T_{2 n+4}}, \\
y_{2 n+1}= & \frac{T_{2 n+1} x_{0} y_{-1} x_{-2}+\left(T_{2 n+1}+T_{2 n}\right) x_{0} y_{-1}+\left(T_{2 n+3}-T_{2 n+2}\right) x_{0}+T_{2 n+2}}{T_{2 n+2} x_{0} y_{-1} x_{-2}+\left(T_{2 n+2}+T_{2 n+1}\right) x_{0} y_{-1}+\left(T_{2 n+4}-T_{2 n+3}\right) x_{0}+T_{2 n+3}}, \\
y_{2 n+2}= & \frac{T_{2 n+2} y_{0} x_{-1} y_{-2}+\left(T_{2 n+2}+T_{2 n+1}\right) y_{0} x_{-1}+\left(T_{2 n+4}-T_{2 n+3}\right) y_{0}+T_{2 n+3}}{T_{2 n+3} y_{0} x_{-1} y_{-2}+\left(T_{2 n+3}+T_{2 n+2}\right) y_{0} x_{-1}+\left(T_{2 n+5}-T_{2 n+4}\right) y_{0}+T_{2 n+4}} .
\end{aligned}
$$

Now, we will study the stability of the equilibrium points of system (3.19) with $a=b=c=d=1$, that is the system

$$
\left\{\begin{array}{l}
x_{n+1}=f_{1}\left(x_{n}, x_{n-1}, x_{n-2}, y_{n}, y_{n-1}, y_{n-2}\right)=\frac{1}{1+y_{n}+x_{n-1} y_{n}+y_{n-2} x_{n-1} y_{n}}, \\
y_{n+1}=f_{2}\left(x_{n}, x_{n-1}, x_{n-2}, y_{n}, y_{n-1}, y_{n-2}\right)=\frac{1}{1+x_{n}+y_{n-1} x_{n}+x_{n-2} y_{n-1} x_{n}} .
\end{array}\right.
$$

For the stability of the equilibrium points, we assume that the initial values are positive real numbers.

The points $\left(\frac{1}{\alpha}, \frac{1}{\alpha}\right),\left(\frac{1}{\beta}, \frac{1}{\beta}\right),\left(\frac{1}{\gamma}, \frac{1}{\gamma}\right)$ and $\left(\frac{1}{\delta}, \frac{1}{\delta}\right)$ are solutions of the system

$$
\left\{\begin{array}{l}
x=\frac{1}{1+y+x y+x y^{2}}, \\
y=\frac{1}{1+x+y x+x^{2} y},
\end{array}\right.
$$

where $\alpha, \beta, \gamma$ and $\delta$ are roots of polynomial characteristic associated to the equation (3.20), see [2]. It follows that $(\bar{x}, \bar{y})=\left(\frac{1}{\alpha}, \frac{1}{\alpha}\right)$ is the only equilibrium point for system $(3.21)$ in $(0,+\infty)^{2}$.

For the equilibrium point $(\bar{x}, \bar{y})=\left(\frac{1}{\alpha}, \frac{1}{\alpha}\right)$, we have the following result. 
Theorem 3. The equilibrium point $(\bar{x}, \bar{y})=\left(\frac{1}{\alpha}, \frac{1}{\alpha}\right)$ is globally asymptotically stable.

Proof. The Jacobean matrix associated to the system (3.21) around the equilibrium point $(\bar{x}, \bar{y})=\left(\frac{1}{\alpha}, \frac{1}{\alpha}\right)$, is given by

$$
A=\left(\begin{array}{cccccc}
0 & -\frac{\alpha+1}{\alpha^{4}} & 0 & -\frac{\alpha-1}{\alpha} & 0 & -\frac{1}{\alpha^{4}} \\
1 & 0 & 0 & 0 & 0 & 0 \\
0 & 1 & 0 & 0 & 0 & 0 \\
-\frac{\alpha-1}{\alpha} & 0 & -\frac{1}{\alpha^{4}} & 0 & -\frac{\alpha+1}{\alpha^{4}} & 0 \\
0 & 0 & 0 & 1 & 0 & 0 \\
0 & 0 & 0 & 0 & 1 & 0
\end{array}\right) .
$$

The characteristic polynomial of $A$ is

$$
P(\lambda)=-h(\lambda) h(-\lambda), \text { where } h(\lambda)=\lambda^{3}-\frac{\alpha-1}{\alpha} \lambda^{2}+\frac{\alpha+1}{\alpha^{4}} \lambda-\frac{1}{\alpha^{4}} .
$$

Consider the two functions

$$
h_{1}(\lambda)=\lambda^{3}, h_{2}(\lambda)=\frac{\alpha-1}{\alpha} \lambda^{2}-\frac{\alpha+1}{\alpha^{4}} \lambda+\frac{1}{\alpha^{4}} .
$$

We have

$$
\left|h_{2}(\lambda)\right| \leq\left|\frac{\alpha-1}{\alpha}\right|+\left|\frac{\alpha+1}{\alpha^{4}}\right|+\left|\frac{1}{\alpha^{4}}\right|<1=\left|h_{1}(\lambda)\right|, \forall \lambda \in \mathbb{C}:|\lambda|=1 .
$$

It follows by Rouché's Theorem, that all the roots of $h(\lambda)$ lie in the open unit disk, and so it is for the roots of $P(\lambda)$. Thus the equilibrium point $\left(\frac{1}{\alpha}, \frac{1}{\alpha}\right)$ is locally asymptotically stable.

It remains to prove that

$$
\lim _{n \rightarrow+\infty} x_{n}=\lim _{n \rightarrow+\infty} y_{n}=\frac{1}{\alpha} .
$$

To this end we will, use the fact

$$
\lim _{n \rightarrow \infty} \frac{T_{n+k}}{T_{n}}=\alpha^{k}, \quad \forall k \in \mathbb{N} .
$$

Using the formula of the solutions, we have

$$
\begin{aligned}
\lim _{n \rightarrow \infty} x_{2 n+1}= & \lim _{n \rightarrow \infty} \frac{T_{2 n+1} y_{0} x_{-1} y_{-2}+\left(T_{2 n+1}+T_{2 n}\right) y_{0} x_{-1}+\left(T_{2 n+3}-T_{2 n+2}\right) y_{0}+T_{2 n+2}}{T_{2 n+2} y_{0} x_{-1} y_{-2}+\left(T_{2 n+2}+T_{2 n+1}\right) y_{0} x_{-1}+\left(T_{2 n+4}-T_{2 n+3}\right) y_{0}+T_{2 n+3}} \\
= & \lim _{n \rightarrow \infty} \frac{\frac{T_{2 n+1}}{T_{2 n}} y_{0} x_{-1} y_{-2}+\left(\frac{T_{2 n+1}}{T_{2 n}}+\frac{T_{2 n}}{T_{2 n}}\right) y_{0} x_{-1}+\left(\frac{T_{2 n+3}}{T_{2 n}}-\frac{T_{2 n+2}}{T_{2 n}}\right) y_{0}+\frac{T_{2 n+2}}{T_{2 n}}}{\frac{T_{2 n}}{T_{2 n}} y_{0} x_{-1} y_{-2}+\left(\frac{T_{2 n+2}}{T_{2 n}}+\frac{T_{2 n+1}}{T_{2 n}}\right) y_{0} x_{-1}+\left(\frac{T_{2 n+4}}{T_{2 n}}-\frac{T_{2 n+3}}{T_{2 n}}\right) y_{0}+\frac{T_{2 n+3}}{T_{2 n}}}
\end{aligned}
$$




$$
\begin{aligned}
& =\frac{\alpha y_{0} x_{-1} y_{-2}+(\alpha+1) y_{0} x_{-1}+\left(\alpha^{3}-\alpha^{2}\right) y_{0}+\alpha^{2}}{\alpha^{2} y_{0} x_{-1} y_{-2}+\left(\alpha^{2}+\alpha^{1}\right) y_{0} x_{-1}+\left(\alpha^{4}-\alpha^{3}\right) y_{0}+\alpha^{3}} \\
& =\frac{1}{\alpha} .
\end{aligned}
$$

Similarly, we show that

$$
\lim _{n \rightarrow \infty} x_{2 n+2}=\lim _{n \rightarrow \infty} y_{2 n+1}=\lim _{n \rightarrow \infty} y_{2 n+2}=\frac{1}{\alpha},
$$

that is

$$
\lim _{n \rightarrow \infty} x_{n}=\lim _{n \rightarrow \infty} y_{n}=\frac{1}{\alpha}
$$

This complete the proof.

As a consequence of Corollary (2), when choosing the initial values to satisfies $y_{-i}=x_{-i}, i=0,1,2$, we get the formulas of the well-defined solutions of the equation

$$
x_{n+1}=\frac{1}{a+b x_{n}+c x_{n-1} x_{n}+d x_{n-2} x_{n-1} x_{n}}, n \in \mathbb{N}_{0} .
$$

These formulas are given in the next result.

Corollary 3. For all $n \in \mathbb{N}_{0}$, the form of every well-defined solution of equation (3.25) is given by

$$
\begin{aligned}
& x_{2 n+1}=\frac{d J_{2 n+1} x_{0} x_{-1} x_{-2}+\left(c J_{2 n+1}+d J_{2 n}\right) x_{0} x_{-1}+\left(J_{2 n+3}-a J_{2 n+2}\right) x_{0}+J_{2 n+2}}{d J_{2 n+2} x_{0} x_{-1} x_{-2}+\left(c J_{2 n+2}+d J_{2 n+1}\right) x_{0} x_{-1}+\left(J_{2 n+4}-a J_{2 n+3}\right) x_{0}+J_{2 n+3}}, \\
& x_{2 n+2}=\frac{d J_{2 n+2} x_{0} x_{-1} x_{-2}+\left(c J_{2 n+2}+d J_{2 n+1}\right) x_{0} x_{-1}+\left(J_{2 n+4}-a J_{2 n+3}\right) x_{0}+J_{2 n+3}}{d J_{2 n+3} x_{0} x_{-1} x_{-2}+\left(c J_{2 n+3}+d J_{2 n+2}\right) x_{0} x_{-1}+\left(J_{2 n+5}-a J_{2 n+4}\right) x_{0}+J_{2 n+4}},
\end{aligned}
$$

which can represented in a unified form as

$$
x_{n+1}=\frac{d J_{n+1} x_{0} x_{-1} x_{-2}+\left(c J_{n+1}+d J_{n}\right) x_{0} x_{-1}+\left(J_{n+3}-a J_{n+2}\right) x_{0}+J_{n+2}}{d J_{n+2} x_{0} x_{-1} x_{-2}+\left(c J_{n+2}+d J_{n+1}\right) x_{0} x_{-1}+\left(J_{n+4}-a J_{n+3}\right) x_{0}+J_{n+3}} .
$$

Moreover, if $a=b=c=d=1$, then (3.25) becomes

$$
x_{n+1}=\frac{1}{1+x_{n}+x_{n-1} x_{n}+x_{n-2} x_{n-1} x_{n}}
$$

and the solutions are expressed in terms of Tetranacci numbers as follows

$$
\begin{aligned}
& x_{2 n+1}=\frac{T_{2 n+1} x_{0} x_{-1} x_{-2}+\left(T_{2 n+1}+T_{2 n}\right) x_{0} x_{-1}+\left(T_{2 n+3}-T_{2 n+2}\right) x_{0}+T_{2 n+2}}{T_{2 n+2} x_{0} x_{-1} x_{-2}+\left(T_{2 n+2}+T_{2 n+1}\right) x_{0} x_{-1}+\left(T_{2 n+4}-T_{2 n+3}\right) x_{0}+T_{2 n+3}}, \\
& x_{2 n+2}=\frac{T_{2 n+2} x_{0} x_{-1} x_{-2}+\left(T_{2 n+2}+T_{2 n+1}\right) x_{0} x_{-1}+\left(T_{2 n+4}-T_{2 n+3}\right) x_{0}+T_{2 n+3}}{T_{2 n+3} x_{0} x_{-1} x_{-2}+\left(T_{2 n+3}+T_{2 n+2}\right) x_{0} x_{-1}+\left(T_{2 n+5}-T_{2 n+4}\right) x_{0}+T_{2 n+4}},
\end{aligned}
$$


or equivalently in the unified form,

$$
x_{n+1}=\frac{T_{n+1} x_{0} x_{-1} x_{-2}+\left(T_{n+1}+T_{n}\right) x_{0} x_{-1}+\left(T_{n+3}-T_{n+2}\right) x_{0}+T_{n+2}}{T_{n+2} x_{0} x_{-1} x_{-2}+\left(T_{n+2}+T_{n+1}\right) x_{0} x_{-1}+\left(T_{n+4}-T_{n+3}\right) x_{0}+T_{n+3}} .
$$

Remark 4. It is not hard to see that $\bar{x}=\frac{1}{\alpha}$ is the unique equilibrium point for (3.27) in $(0,+\infty)$ when taking the initial values positive real numbers.

The linearized equation of (3.27) about the equilibrium point $\bar{x}=\frac{1}{\alpha}$ is

$$
w_{n+1}=-\frac{\alpha-1}{\alpha} w_{n}-\frac{\alpha+1}{\alpha^{4}} w_{n-1}-\frac{1}{\alpha^{4}} w_{n-2} .
$$

We have $\frac{\alpha-1}{\alpha}, \frac{\alpha+1}{\alpha^{4}}, \frac{1}{\alpha^{4}}$ are real numbers and

$$
\left|\frac{\alpha-1}{\alpha}\right|+\left|\frac{\alpha+1}{\alpha^{4}}\right|+\left|\frac{1}{\alpha^{4}}\right|<1,
$$

thus, $\bar{x}=\frac{1}{\alpha}$ is locally asymptotically stable.

Now, using (3.23) and (3.30), we get

$$
\begin{aligned}
\lim _{n \rightarrow \infty} x_{n+1} & =\lim _{n \rightarrow \infty} \frac{T_{n+1} x_{0} x_{-1} x_{-2}+\left(T_{n+1}+T_{n}\right) x_{0} x_{-1}+\left(T_{n+3}-T_{n+2}\right) x_{0}+T_{n+2}}{T_{n+2} x_{0} x_{-1} x_{-2}+\left(T_{n+2}+T_{n+1}\right) x_{0} x_{-1}+\left(T_{n+4}-T_{n+3}\right) x_{0}+T_{n+3}} \\
& =\lim _{n \rightarrow \infty} \frac{\frac{T_{n+1}}{T_{n}} x_{0} x_{-1} x_{-2}+\left(\frac{T_{n+1}}{T_{n}}+\frac{T_{n}}{T_{n}}\right) x_{0} x_{-1}+\left(\frac{T_{n+3}}{T_{n}}-\frac{T_{n+2}}{T_{n}}\right) x_{0}+\frac{T_{n+2}}{T_{n}}}{T_{n} x_{-1} x_{-2}+\left(\frac{T_{n+2}}{T_{n}}+\frac{T_{n+1}}{T_{n}}\right) x_{0} x_{-1}+\left(\frac{T_{n+4}}{T_{n}}-\frac{T_{n+3}}{T_{n}}\right) x_{0}+\frac{T_{n+3}}{T_{n}}} \\
& =\frac{\alpha x_{0} x_{-1} x_{-2}+(\alpha+1) x_{0} x_{-1}+\left(\alpha^{3}-\alpha^{2}\right) x_{0}+\alpha^{2}}{\alpha^{2} x_{0} x_{-1} x_{-2}+\left(\alpha^{2}+\alpha\right) x_{0} x_{-1}+\left(\alpha^{4}-\alpha^{3}\right) x_{0}+\alpha^{3}} \\
& =\frac{1}{\alpha} .
\end{aligned}
$$

In summary, the equilibrium point $\bar{x}=\frac{1}{\alpha}$ is globally asymptotically stable.

\subsection{Particular cases of system (3.1)}

3.2.1. The case $d=0$ and $c \neq 0$

In this case the system (3.1) take the form:

$$
\left\{\begin{array}{l}
x_{n+1}=f^{-1}\left[a+\frac{b}{g\left(y_{n}\right)}+\frac{c}{g\left(y_{n}\right) f\left(x_{n-1}\right)}\right], \\
y_{n+1}=g^{-1}\left[a+\frac{b}{f\left(x_{n}\right)}+\frac{c}{f\left(x_{n}\right) g\left(y_{n-1}\right)}\right], n \in \mathbb{N}_{0} .
\end{array}\right.
$$


Corollary 4. The formulas of well-defined solutions of system (3.32), are given, for all $n \in \mathbb{N}_{0}$, by

$$
\begin{aligned}
& x_{2 n+1}=f^{-1}\left[\frac{c \widetilde{J}_{2 n+1}+\left(\widetilde{J}_{2 n+3}-a \widetilde{J}_{2 n+2}\right) f\left(x_{-1}\right)+\widetilde{J}_{2 n+2} g\left(y_{0}\right) f\left(x_{-1}\right)}{c \widetilde{J}_{2 n}+\left(\widetilde{J}_{2 n+2}-a \widetilde{J}_{2 n+1}\right) f\left(x_{-1}\right)+\widetilde{J}_{2 n+1} g\left(y_{0}\right) f\left(x_{-1}\right)}\right], \\
& x_{2 n+2}=f^{-1}\left[\frac{c \widetilde{J}_{2 n+2}+\left(\widetilde{J}_{2 n+4}-a \widetilde{J}_{2 n+3}\right) g\left(y_{-1}\right)+\widetilde{J}_{2 n+3} f\left(x_{0}\right) g\left(y_{-1}\right)}{c \widetilde{J}_{2 n+1}+\left(\widetilde{J}_{2 n+3}-a \widetilde{J}_{2 n+2}\right) g\left(y_{-1}\right)+\widetilde{J}_{2 n+2} f\left(x_{0}\right) g\left(y_{-1}\right)}\right], \\
& y_{2 n+1}=g^{-1}\left[\frac{c \widetilde{J}_{2 n+1}+\left(\widetilde{J}_{2 n+3}-a \widetilde{J}_{2 n+2}\right) g\left(y_{-1}\right)+\widetilde{J}_{2 n+2} f\left(x_{0}\right) g\left(y_{-1}\right)}{c \widetilde{J}_{2 n}+\left(\widetilde{J}_{2 n+2}-a \widetilde{J}_{2 n+1}\right) g\left(y_{-1}\right)+\widetilde{J}_{2 n+1} f\left(x_{0}\right) g\left(y_{-1}\right)}\right], \\
& y_{2 n+2}=g^{-1}\left[\frac{c \widetilde{J}_{2 n+2}+\left(\widetilde{J}_{2 n+4}-a \widetilde{J}_{2 n+3}\right) f\left(x_{-1}\right)+\widetilde{J}_{2 n+3} g\left(y_{0}\right) f\left(x_{-1}\right)}{c \widetilde{J}_{2 n+1}+\left(\widetilde{J}_{2 n+3}-a \widetilde{J}_{2 n+2}\right) f\left(x_{-1}\right)+\widetilde{J}_{2 n+2} g\left(y_{0}\right) f\left(x_{-1}\right)}\right] .
\end{aligned}
$$

If $g=f$ and $y_{-i}=x_{-i}, i=0,1,2$ then, system (3.32) becomes

$$
x_{n+1}=f^{-1}\left[a+\frac{b}{f\left(x_{n}\right)}+\frac{c}{f\left(x_{n}\right) f\left(x_{n-1}\right)}\right], n \in \mathbb{N}_{0},
$$

hence, every well-defined solution of equation (3.33) is given by

$$
\begin{aligned}
& x_{2 n+1}=f^{-1}\left[\frac{c \widetilde{J}_{2 n+1}+\left(\widetilde{J}_{2 n+3}-a \widetilde{J}_{2 n+2}\right) f\left(x_{-1}\right)+\widetilde{J}_{2 n+2} f\left(x_{0}\right) f\left(x_{-1}\right)}{c \widetilde{J}_{2 n}+\left(\widetilde{J}_{2 n+2}-a \widetilde{J}_{2 n+1}\right) f\left(x_{-1}\right)+\widetilde{J}_{2 n+1} f\left(x_{0}\right) f\left(x_{-1}\right)}\right], \\
& x_{2 n+2}=f^{-1}\left[\frac{c \widetilde{J}_{2 n+2}+\left(\widetilde{J}_{2 n+4}-a \widetilde{J}_{2 n+3}\right) f\left(x_{-1}\right)+\widetilde{J}_{2 n+3} f\left(x_{0}\right) f\left(x_{-1}\right)}{c \widetilde{J}_{2 n+1}+\left(\widetilde{J}_{2 n+3}-a \widetilde{J}_{2 n+2}\right) f\left(x_{-1}\right)+\widetilde{J}_{2 n+2} f\left(x_{0}\right) f\left(x_{-1}\right)}\right],
\end{aligned}
$$

or in the unified form

$$
x_{n+1}=f^{-1}\left[\frac{c \widetilde{J}_{n+1}+\left(\widetilde{J}_{n+3}-a \widetilde{J}_{n+2}\right) f\left(x_{-1}\right)+\widetilde{J}_{n+2} f\left(x_{0}\right) f\left(x_{-1}\right)}{c \widetilde{J}_{n}+\left(\widetilde{J}_{n+2}-a \widetilde{J}_{n+1}\right) f\left(x_{-1}\right)+\widetilde{J}_{n+1} f\left(x_{0}\right) f\left(x_{-1}\right)}\right], n \in \mathbb{N}_{0},
$$

where $\left(\widetilde{J}_{n}\right)_{n \geq 0}$ is the sequence defined by

$$
\widetilde{J}_{n+3}=a \widetilde{J}_{n+2}+b \widetilde{J}_{n+1}+c \widetilde{J}_{n}, \widetilde{J}_{0}=0, \widetilde{J}_{1}=1, \widetilde{J}_{2}=a, n \in \mathbb{N}_{0} .
$$

3.2.2. The system $x_{n+1}=\frac{1}{a+b y_{n}+c x_{n-1} y_{n}}, y_{n+1}=\frac{1}{a+b x_{n}+c y_{n-1} x_{n}}$

In the following, we are interested in a particular system of (3.32) and some particular equations of its one dimensional version. 
Let $f(t)=\frac{1}{t}$ and $g(t)=\frac{1}{t}$, then, system (3.32) becomes

$$
x_{n+1}=\frac{1}{a+b y_{n}+c x_{n-1} y_{n}}, y_{n+1}=\frac{1}{a+b x_{n}+c y_{n-1} x_{n}} .
$$

So, by Corollary 4, we get the following result.

Corollary 5. For all $n \in \mathbb{N}_{0}$, the representation of well-defined solution of system (3.34) is

$$
\begin{array}{r}
x_{2 n+1}=\frac{c \widetilde{J}_{2 n} y_{0} x_{-1}+\left(\widetilde{J}_{2 n+2}-a \widetilde{J}_{2 n+1}\right) y_{0}+\widetilde{J}_{2 n+1}}{c \widetilde{J}_{2 n+1} y_{0} x_{-1}+\left(\widetilde{J}_{2 n+3}-a \widetilde{J}_{2 n+2}\right) y_{0}+\widetilde{J}_{2 n+2}}, \\
x_{2 n+2}=\frac{c \widetilde{J}_{2 n+1} x_{0} y_{-1}+\left(\widetilde{J}_{2 n+3}-a \widetilde{J}_{2 n+2}\right) x_{0}+\widetilde{J}_{2 n+2}}{c \widetilde{J}_{2 n+2} x_{0} y_{-1}+\left(\widetilde{J}_{2 n+4}-a \widetilde{J}_{2 n+3}\right) x_{0}+\widetilde{J}_{2 n+3}}, \\
y_{2 n+1}=\frac{c \widetilde{J}_{2 n} x_{0} y_{-1}+\left(\widetilde{J}_{2 n+2}-a \widetilde{J}_{2 n+1}\right) x_{0}+\widetilde{J}_{2 n+1}}{c \widetilde{J}_{2 n+1} x_{0} y_{-1}+\left(\widetilde{J}_{2 n+3}-a \widetilde{J}_{2 n+2}\right) x_{0}+\widetilde{J}_{2 n+2}}, \\
y_{2 n+2}=\frac{c \widetilde{J}_{2 n+1} y_{0} x_{-1}+\left(\widetilde{J}_{2 n+3}-a \widetilde{J}_{2 n+2}\right) y_{0}+\widetilde{J}_{2 n+2}}{c \widetilde{J}_{2 n+2} y_{0} x_{-1}+\left(\widetilde{J}_{2 n+4}-a \widetilde{J}_{2 n+3}\right) y_{0}+\widetilde{J}_{2 n+3}} .
\end{array}
$$

Moreover, the solutions of the following equation

$$
x_{n+1}=\frac{1}{a+b x_{n}+c x_{n-1} x_{n}},
$$

follows directly from those of the system (3.34) by taking $y_{-i}=x_{-i}, i=0,1$. Then, representation of well-defined solution is

$$
\begin{gathered}
x_{2 n+1}=\frac{c \widetilde{J}_{2 n} x_{0} x_{-1}+\left(\widetilde{J}_{2 n+2}-a \widetilde{J}_{2 n+1}\right) x_{0}+\widetilde{J}_{2 n+1}}{c \widetilde{J}_{2 n+1} x_{0} x_{-1}+\left(\widetilde{J}_{2 n+3}-a \widetilde{J}_{2 n+2}\right) x_{0}+\widetilde{J}_{2 n+2}}, \\
x_{2 n+2}=\frac{c \widetilde{J}_{2 n+1} x_{0} x_{-1}+\left(\widetilde{J}_{2 n+3}-a \widetilde{J}_{2 n+2}\right) x_{0}+\widetilde{J}_{2 n+2}}{c \widetilde{J}_{2 n+2} x_{0} x_{-1}+\left(\widetilde{J}_{2 n+4}-a \widetilde{J}_{2 n+3}\right) x_{0}+\widetilde{J}_{2 n+3}} .
\end{gathered}
$$

Recently in [12], the authors studied the particular difference equations

$$
\begin{gathered}
x_{n+1}=\frac{1}{-1+x_{n}+x_{n-1} x_{n}}, \\
x_{n+1}=\frac{1}{1+x_{n}-x_{n-1} x_{n}},
\end{gathered}
$$




$$
\begin{gathered}
x_{n+1}=\frac{1}{1-x_{n}+x_{n-1} x_{n}}, \\
x_{n+1}=\frac{1}{-1-x_{n}-x_{n-1} x_{n}},
\end{gathered}
$$

and as a generalization of (3.38), (3.39), (3.40), (3.41) the authors studied again in [11], the equation

$$
x_{n+1}=\frac{1}{\frac{\beta}{\gamma}+\frac{\alpha}{\gamma} x_{n}+\frac{1}{\gamma} x_{n-1} x_{n}}, \gamma \neq 0 .
$$

Clearly these equations are particular cases of equation (3.35).

In fact to obtain equation (3.38) it suffices to take $a=-1, b=1$ and $c=1$ in (3.35), when choosing $a=1, b=1$ and $c=-1$ in (3.35) we get equation (3.39). Equation (3.40) follows from (3.35) for the choice $a=1, b=-1$ and $c=1$. Again if we take $a=-1, b=-1$ and $c=-1$ in (3.35) we get the equation (3.41), finally for the choice $a=\frac{\beta}{\gamma}, b=\frac{\alpha}{\gamma}$ and $c=\frac{1}{\gamma}$ in (3.35) we find the equation (3.42).

Noting, that the authors studied in [13] the following systems

$$
\begin{gathered}
\left\{\begin{array}{l}
x_{n+1}=\frac{1}{1+y_{n}+x_{n-1} y_{n}}, \\
y_{n+1}=\frac{1}{1+x_{n}+y_{n-1} x_{n}},
\end{array}\right. \\
\left\{\begin{array}{l}
x_{n+1}=\frac{1}{-1+y_{n}-x_{n-1} y_{n}}, \\
y_{n+1}=\frac{1}{-1+x_{n}-y_{n-1} x_{n}} .
\end{array}\right.
\end{gathered}
$$

Systems (3.43) and (3.44) are particular cases of system (3.34) for the choices $a=$ $b=c=1$ and $a=-1, b=1, c=-1$ respectively.

Now, we will investigate each of these cases separately.

(1) Consider $a=-1, b=1, c=1$, equation (3.35) becomes (3.38). For this choice of the parameters, we get the sequence $\left(\widetilde{J}_{n}\right)_{n=0}^{\infty}$ defined by:

$$
\widetilde{J}_{n+3}=-\widetilde{J}_{n+2}+\widetilde{J}_{n+1}+\widetilde{J}_{n}, \widetilde{J}_{0}=0, \widetilde{J}_{1}=1, \widetilde{J}_{2}=-1 .
$$

It is easy to see that

$$
\widetilde{J_{n}}=-\left(\frac{1}{4}+\frac{1}{2} n\right)(-1)^{n}+\frac{1}{4}, \quad n \in \mathbb{N}_{0} .
$$

From this it follows that

$$
\widetilde{J}_{2 n}=-n, \widetilde{J}_{2 n+1}=n+1 .
$$

Replacing in (3.36) and (3.37), we obtain

$$
x_{2 n+1}=\frac{-n x_{-1} x_{0}+n+1}{(n+1) x_{-1} x_{0}+x_{0}-(n+1)},
$$




$$
x_{2 n+2}=\frac{(n+1) x_{0} x_{-1}+x_{0}-(n+1)}{-(n+1) x_{0} x_{-1}+n+2},
$$

and these are the formulas given in [12] for the solutions of (3.38).

(2) Consider $a=1, b=1, c=-1$, equation (3.35) becomes (3.39). For this choice of the parameters, we get the sequence $\left(\widetilde{J}_{n}\right)_{n=0}^{\infty}$ defined by:

$$
\widetilde{J}_{n+3}=\widetilde{J}_{n+2}+\widetilde{J}_{n+1}-\widetilde{J}_{n}, \widetilde{J}_{0}=0, \widetilde{J}_{1}=1, \widetilde{J}_{2}=1 .
$$

It is easy to see that

$$
\widetilde{J}_{n}=\frac{1}{4}+\frac{1}{2} n-\frac{1}{4}(-1)^{n}, \quad n \in \mathbb{N}_{0} .
$$

From this it follows that

$$
\widetilde{J}_{2 n}=n, \widetilde{J}_{2 n+1}=n+1 .
$$

Replacing in (3.36) and (3.37), we get

$$
\begin{aligned}
& x_{2 n+1}=\frac{n x_{-1} x_{0}-(n+1)}{(n+1) x_{-1} x_{0}-x_{0}-(n+1)}, \\
& x_{2 n+2}=\frac{(n+1) x_{0} x_{-1}-x_{0}-(n+1)}{(n+1) x_{0} x_{-1}-(n+2)},
\end{aligned}
$$

and these are the formulas given in [12] for the solutions of (3.39).

(3) Consider $a=1, b=-1, c=1$, equation (3.35) becomes (3.40). For this choice of the parameters, we get the sequence $\left(\widetilde{J}_{n}\right)_{n=0}^{\infty}$ defined by:

$$
\widetilde{J}_{n+3}=\widetilde{J}_{n+2}-\widetilde{J}_{n+1}+\widetilde{J}_{n}, \widetilde{J}_{0}=0, \widetilde{J}_{1}=1, \widetilde{J}_{2}=1 .
$$

We have

$$
\begin{aligned}
\widetilde{J}_{n} & =\frac{1}{2}+\frac{1}{2(i-1)}(i)^{n}+\frac{-1}{2(i+1)}(-i)^{n} \\
& =\frac{1}{2}+\left(\frac{-1}{4}-\frac{1}{4} i\right)(i)^{n}+\left(\frac{-1}{4}+\frac{1}{4} i\right)(-i)^{n}, \quad n \in \mathbb{N}_{0} .
\end{aligned}
$$

From this it follows that

$$
\widetilde{J}_{2 n}=\frac{1}{2}-\frac{1}{2}(-1)^{n}, \widetilde{J}_{2 n+1}=\frac{1}{2}+\frac{1}{2}(-1)^{n},
$$

depending on $n$ is even or odd, we get

$$
\widetilde{J}_{4 n}=0, \widetilde{J}_{4 n+1}=1, \widetilde{J}_{4 n+2}=1, \widetilde{J}_{4 n+3}=0 .
$$

Replacing in (3.36) and (3.37), we obtain

$$
x_{4 n+1}=\frac{1}{x_{-1} x_{0}-x_{0}+1},
$$




$$
\begin{aligned}
& x_{4 n+2}=\frac{x_{-1} x_{0}-x_{0}+1}{x_{-1} x_{0}}, \\
& x_{4 n+3}=x_{-1}, \\
& x_{4 n+4}=x_{0},
\end{aligned}
$$

and these are the formulas given in [12] for the solutions of (3.39).

(4) Consider $a=b=c=-1$, equation (3.35) becomes (3.41). For this choice of the parameters, we get the sequence $\left(\widetilde{J}_{n}\right)_{n=0}^{\infty}$ defined by:

$$
\widetilde{J}_{n+3}=-\widetilde{J}_{n+2}-\widetilde{J}_{n+1}-\widetilde{J}_{n}, \widetilde{J}_{0}=0, \widetilde{J}_{1}=1, \widetilde{J}_{2}=-1 .
$$

We have

$$
\begin{aligned}
\widetilde{J}_{n} & =\frac{-1}{2}(-1)^{n}+\frac{1}{2(1+i)}(i)^{n}+\frac{1}{2(1-i)}(-i)^{n} \\
& =\frac{-1}{2}(-1)^{n}+\left(\frac{1}{4}-\frac{1}{4} i\right)(i)^{n}+\left(\frac{1}{4}+\frac{1}{4} i\right)(-i)^{n}, \quad n \in \mathbb{N}_{0} .
\end{aligned}
$$

From this it follows that

$$
\widetilde{J}_{2 n}=\frac{-1}{2}+\frac{1}{2}(-1)^{n}, \widetilde{J}_{2 n+1}=\frac{1}{2}+\frac{1}{2}(-1)^{n},
$$

depending on $n$ is even or odd, we get

$$
\widetilde{J}_{4 n}=0, \widetilde{J}_{4 n+1}=1, \widetilde{J}_{4 n+2}=-1, \widetilde{J}_{4 n+3}=0 .
$$

Replacing in (3.36) and (3.37), we get

$$
\begin{aligned}
& x_{4 n+1}=\frac{1}{-x_{-1} x_{0}-x_{0}-1}, \\
& x_{4 n+2}=\frac{-x_{-1} x_{0}-x_{0}-1}{x_{-1} x_{0}}, \\
& x_{4 n+3}=x_{-1}, \\
& x_{4 n+4}=x_{0},
\end{aligned}
$$

and these are the formulas given in [12] for the solutions of (3.41).

(5) Consider $a=b=c=1$, system (3.34) becomes (3.43). For this choice of the parameters, the sequence $\left(\widetilde{J}_{n}\right)_{n=0}^{+\infty}$ will be nothing other than the famous Tribonacci sequence defined for $n \in \mathbb{N}_{0}$ by

$$
T_{n+3}=T_{n+2}+T_{n+1}+T_{n}, T_{0}=0, \quad T_{1}=T_{2}=1 .
$$


The solutions are given by

$$
\left\{\begin{array}{l}
x_{2 n+1}=\frac{T_{2 n} y_{0} x_{-1}+\left(T_{2 n+2}-T_{2 n+1}\right) y_{0}+T_{2 n+1}}{T_{2 n+1} y_{0} x_{-1}+\left(T_{2 n+3}-T_{2 n+2}\right) y_{0}+T_{2 n+2}}, \\
x_{2 n+2}=\frac{T_{2 n+1} x_{0} y_{-1}+\left(T_{2 n+3}-T_{2 n+2}\right) x_{0}+T_{2 n+2}}{T_{2 n+2} x_{0} y_{-1}+\left(T_{2 n+4}-T_{2 n+3}\right) x_{0}+T_{2 n+3}}, \\
y_{2 n+1}=\frac{T_{2 n} x_{0} y_{-1}+\left(T_{2 n+2}-T_{2 n+1}\right) x_{0}+T_{2 n+1}}{T_{2 n+1} x_{0} y_{-1}+\left(T_{2 n+3}-T_{2 n+2}\right) x_{0}+T_{2 n+2}}, \\
y_{2 n+2}=\frac{T_{2 n+1} y_{0} x_{-1}+\left(T_{2 n+3}-T_{2 n+2}\right) y_{0}+T_{2 n+2}}{T_{2 n+2} y_{0} x_{-1}+\left(T_{2 n+4}-T_{2 n+3}\right) y_{0}+T_{2 n+3}} .
\end{array}\right.
$$

(6) Consider $a=-1, b=1, c=-1$, system (3.34) becomes (3.44). For this choice of the parameters, we get the sequence $\left(\widetilde{J}_{n}\right)_{n=0}^{\infty}$ defined by:

$$
\widetilde{J}_{n+3}=-\widetilde{J}_{n+2}+\widetilde{J}_{n+1}-\widetilde{J}_{n}, \widetilde{J}_{0}=0, \widetilde{J}_{1}=1, \widetilde{J}_{2}=-1 .
$$

Using the fact that $\widetilde{J}_{n}=(-1)^{n+1} T_{n}$ ([1]), we get from Corollary 5 that the representation of well-defined solutions of system (3.44) is

$$
\left\{\begin{array}{l}
x_{2 n+1}=\frac{-\left(T_{2 n} y_{0} x_{-1}+\left(T_{2 n+1}-T_{2 n+2}\right) y_{0}+T_{2 n+1}\right)}{T_{2 n+1} y_{0} x_{-1}-\left(T_{2 n+1}+T_{2 n}\right) y_{0}+T_{2 n+2}}, \\
x_{2 n+2}=\frac{-\left(T_{2 n+1} x_{0} y_{-1}+\left(T_{2 n+2}-T_{2 n+3}\right) x_{0}+T_{2 n+2}\right)}{T_{2 n+2} x_{0} y_{-1}-\left(T_{2 n+2}+T_{2 n+1}\right) x_{0}+T_{2 n+3}}, \\
y_{2 n+1}=\frac{-\left(T_{2 n} x_{0} y_{-1}+\left(T_{2 n+1}-T_{2 n+2}\right) x_{0}+T_{2 n+1}\right)}{T_{2 n+1} x_{0} y_{-1}-\left(T_{2 n+1}+T_{2 n}\right) x_{0}+T_{2 n+2}} \\
y_{2 n+2}=\frac{-\left(T_{2 n+1} y_{0} x_{-1}+\left(T_{2 n+2}-T_{2 n+3}\right) y_{0}+T_{2 n+2}\right)}{T_{2 n+2} y_{0} x_{-1}-\left(T_{2 n+2}+T_{2 n+1}\right) y_{0}+T_{2 n+3}}
\end{array}\right.
$$

and these are the formulas given in [13] for the solutions of (3.44).

3.2.3. Case $d=0, c \neq 0$ and $a=0$

In this case, we obtain the following system

$$
\left\{\begin{array}{l}
x_{n+1}=f^{-1}\left[\frac{b}{g\left(y_{n}\right)}+\frac{c}{g\left(y_{n}\right) f\left(x_{n-1}\right)}\right], \\
y_{n+1}=g^{-1}\left[\frac{b}{f\left(x_{n}\right)}+\frac{c}{f\left(x_{n}\right) g\left(y_{n-1}\right)}\right], n \in \mathbb{N}_{0} .
\end{array}\right.
$$

Here, $\left(\widetilde{J}_{n}\right)_{n \geq 0}$ will be the sequence $\left(\mathscr{P}_{n}\right)_{n \geq 0}$ defined by

$$
\mathcal{P}_{n+3}=b \mathcal{P}_{n+1}+c \mathcal{P}_{n}, \mathcal{P}_{0}=0, \mathcal{P}_{1}=1, \mathcal{P}_{2}=0, n \in \mathbb{N}_{0} .
$$

So, well-defined solutions are expressed in terms of $\left(\mathcal{P}_{n}\right)_{n \geq 0}$ and takes the form

$$
\begin{aligned}
& x_{2 n+1}=f^{-1}\left[\frac{c \mathcal{P}_{2 n+1}+\mathcal{P}_{2 n+3} f\left(x_{-1}\right)+\mathcal{P}_{2 n+2} g\left(y_{0}\right) f\left(x_{-1}\right)}{c \mathcal{P}_{2 n}+\mathcal{P}_{2 n+2} f\left(x_{-1}\right)+\mathcal{P}_{2 n+1} g\left(y_{0}\right) f\left(x_{-1}\right)}\right], n \in \mathbb{N}_{0}, \\
& x_{2 n+2}=f^{-1}\left[\frac{c \mathcal{P}_{2 n+2}+\mathcal{P}_{2 n+4} g\left(y_{-1}\right)+\mathcal{P}_{2 n+3} f\left(x_{0}\right) g\left(y_{-1}\right)}{c \mathcal{P}_{2 n+1}+\mathcal{P}_{2 n+3} g\left(y_{-1}\right)+\mathcal{P}_{2 n+2} f\left(x_{0}\right) g\left(y_{-1}\right)}\right], n \in \mathbb{N}_{0},
\end{aligned}
$$




$$
\begin{aligned}
& y_{2 n+1}=g^{-1}\left[\frac{c \mathcal{P}_{2 n+1}+\mathcal{P}_{2 n+3} g\left(y_{-1}\right)+\mathcal{P}_{2 n+2} f\left(x_{0}\right) g\left(y_{-1}\right)}{c \mathcal{P}_{2 n}+\mathcal{P}_{2 n+2} g\left(y_{-1}\right)+\mathcal{P}_{2 n+1} f\left(x_{0}\right) g\left(y_{-1}\right)}\right], n \in \mathbb{N}_{0}, \\
& y_{2 n+2}=g^{-1}\left[\frac{c \mathcal{P}_{2 n+2}+\mathcal{P}_{2 n+4} f\left(x_{-1}\right)+\mathcal{P}_{2 n+3} g\left(y_{0}\right) f\left(x_{-1}\right)}{c \mathcal{P}_{2 n+1}+\mathcal{P}_{2 n+3} f\left(x_{-1}\right)+\mathcal{P}_{2 n+2} g\left(y_{0}\right) f\left(x_{-1}\right)}\right], n \in \mathbb{N}_{0} .
\end{aligned}
$$

for system (3.48) and by

$$
x_{n+1}=f^{-1}\left[\frac{c \mathcal{P}_{n+1}+\mathcal{P}_{n+3} f\left(x_{-1}\right)+\mathcal{P}_{n+2} f\left(x_{0}\right) f\left(x_{-1}\right)}{c \mathcal{P}_{n}+\mathcal{P}_{n+2} f\left(x_{-1}\right)+\mathcal{P}_{n+1} f\left(x_{0}\right) f\left(x_{-1}\right)}\right], n \in \mathbb{N}_{0},
$$

for its one dimensional version, that is the equation

$$
x_{n+1}=f^{-1}\left[\frac{b}{f\left(x_{n}\right)}+\frac{c}{f\left(x_{n}\right) f\left(x_{n-1}\right)}\right], n \in \mathbb{N}_{0} .
$$

Also, (3.48) generalize some works in the literature, see, for example [5] and [21].

3.2.4. Case $c=d=0, b \neq 0$

In this case, we get the system

$$
x_{n+1}=f^{-1}\left[a+\frac{b}{g\left(y_{n}\right)}\right], y_{n+1}=g^{-1}\left[a+\frac{b}{f\left(x_{n}\right)}\right], n \in \mathbb{N}_{0} .
$$

In this case, the well-defined solutions will be expressed using terms of the sequence $\left(\widetilde{F}_{n}\right)_{n \geq 0}=\left(\widetilde{J}_{n+1}\right)_{n \geq 0}$, defined by

$$
\widetilde{F}_{n+2}=a \widetilde{F}_{n+1}+b \widetilde{F}_{n}, \widetilde{F}_{0}=1, \widetilde{F}_{1}=a, n \in \mathbb{N}_{0}
$$

and the solutions for (3.50) and its one dimensional version, are obtained from Corollary (4), by writing $\widetilde{F}_{n-1}$ instead of $\widetilde{J}_{n}$. System (3.48) and its one dimensional version, generalize some existing works, for example [17], [18].

Remark 5. If $b=c=d=0, a \neq 0$, we get

$$
x_{n}=f^{-1}(a), y_{n}=g^{-1}(a), n=1,2, \cdots \text {. }
$$

\section{CONCLUSiON}

In this work, we have presented formulas of well-defined solutions of some general systems of difference equations defined by one to one functions on a set $D$ of real numbers. Noting that the obtained formulas of the solutions of our systems are expressed using some remarkable sequences, like Fibonacci, Tribonaci, Padovan, Tetranacci and their generalizations. Our work generalizes a lot of existing works in the literature on solvable difference equations and systems. Although, the condition "one to one functions", reduces the set of possibilities surveyed by this work. But our results, can be used to obtain the formulas of well-defined solutions of other systems, that their solvability, seems for the first sight impossible. So, under appropriate 
choice of the the set $D$, we can solve complicated difference equations and systems involving for example functions like "tan", "In" and others.

\section{ACKNOWLEDGMENTS}

The authors would like to thank the anonymous referee for his(her) remarks and suggestions. Y. Akrour and N. Touafek acknowledge the support of DGRSDTMESRS (DZ).

\section{REFERENCES}

[1] Y. Akrour, N. Touafek, and Y. Halim, "On a system of difference equations of second order solved in closed form," Miskolc Math. Notes, vol. 20, no. 2, pp. 701-717, 2019, doi: 10.18514/MMN.2019.2923.

[2] Y. Akrour, N. Touafek, and Y. Halim, "On a system of difference equations of third order solved in closed form," preprint: arXiv, vol. 1910.14365v2, 2019.

[3] R. Azizi, "Global behaviour of the rational Riccati difference equation of order two: the general case," J. Difference Equ. Appl., vol. 18, no. 6, pp. 947-961, 2012, doi: 10.1080/10236198.2010.532790.

[4] R. Azizi, "Global behavior of the higher order rational riccati difference equation," J. Difference Equ. Appl., vol. 230, pp. 1-8, 2014, doi: 10.1016/j.amc.2013.12.055.

[5] Y. Halim and J. F. T. Rabago, "On the solutions of a second-order difference equation in terms of generalized padovan sequences," Math. Slovaca, vol. 68, no. 3, pp. 625-638, 2018, doi: 10.1515/ms-2017-0130.

[6] G. S. Hathiwala and D. V. Shah, "Binet-type formula for the sequence of tetranacci numbers by alternate methods," Math. J. Interdiscip. Sci., vol. 6, no. 1, pp. 37-48, 2017, doi: 10.15415/mjis.2017.61004.

[7] M. Kara, N. Touafek, and Y. Yazlik, "Well-defined solutions of a three-dimensional system of difference equations," Gazi University Journal of Science, vol. 33, pp. 767 - 778, 2020, doi: 10.35378/gujs.641441.

[8] M. Kara and Y. Yazlik, "Solvability of a system of nonlinear difference equations of higher order," Turk. J. Math., vol. 43, no. 3, pp. 1533-1565, 2019, doi: 10.3906/mat-1902-24.

[9] M. Kara and Y. Yazlik, "On the system of difference equations $x_{n}=\frac{x_{n-2} y_{n-3}}{y_{n-1}\left(a_{n}+b_{n} x_{n-2} y_{n-3}\right)}, y_{n}=$ $\frac{y_{n-2} x_{n-3}}{x_{n-1}\left(\alpha_{n}+\beta_{n} y_{n-2} x_{n-3}\right)}$, J. Math. Ext., vol. 14, no. 1, pp. 41-59, 2020.

[10] M. Kara, Y. Yazlik, and D. T. Tollu, "Solvability of a system of higher order nonlinear difference equations,” Hacet. J. Math. Stat., vol. 49, pp. 1566 - 1593, 2020, doi: 10.15672/hujms.474649.

[11] I. Okumuş and Y. Soykan, "On the dynamics of solutions of a rational difference equation via generalized tribonacci numbers," arXiv preprint arXiv:1906.11629, 2019.

[12] I. Okumuş and Y. Soykan, "On the solutions of four second-order nonlinear difference equations," Univers. J. Math. Appl., vol. 2, no. 3, pp. 116-125, 2019, doi: 10.32323/ujma.589274.

[13] I. Okumuş and Y. Soykan, "On the solutions of systems of difference equations via tribonacci numbers," arXiv preprint arXiv:1906.09987, 2019.

[14] S. Stevic, "Some representations of the general solution to a difference equation of additive type," Adv. Difference Equ., vol. 2019, no. 1, p. 431, 2019, doi: 10.1186/s13662-019-2365-0.

[15] S. Stević, B. Iričanin, and W. Kosmala, "Representations of general solutions to some classes of nonlinear difference equations," Adv. Difference Equ., vol. 2019, no. 1, pp. 1-21, 2019, doi: 10.1186/s13662-019-2013-8. 
[16] S. Stevic, B. Iricanin, W. Kosmala, and Z. Smarda, "Representation of solutions of a solvable nonlinear difference equation of second order," Electron. J. Qual. Theory Differ. Equ., no. 95, pp. 1-18, 2018, doi: 10.14232/ejqtde.2018.1.95.

[17] D. T. Tollu, Y. Yazlik, and N. Taskara, "On the solutions of two special types of Riccati difference equation via Fibonacci numbers," Adv. Difference Equ., vol. 174, no. 1, pp. 1-7, 2013, doi: 10.1186/1687-1847-2013-174.

[18] D. T. Tollu, Y. Yazlik, and N. Taskara, "The solutions of four Riccati difference equations associated with Fibonacci numbers," Balkan J. Math., vol. 2, no. 1, pp. 163-172, 2014.

[19] M. E. Waddill, “The tetranacci sequence and generalizations," Fibonacci Q., vol. 30, no. 1, pp. 9-20, 1992.

[20] Y. Yazlik and M. Kara, "On a solvable system of difference equations of fifth-order," Eskisehir Tech. Univ. J. Sci. Tech. B-Theoret. Sci., vol. 7, no. 1, pp. 29-45, 2019, doi: 10.20290/aubtdb.422910.

[21] Y. Yazlik, D. T. Tollu, and N. Taskara, "On the solutions of difference equation systems with Padovan numbers," Appl. Math., vol. 12, no. 1, pp. 15-202, 2013, doi: 10.4236/am.2013.412A1002.

[22] Y. Yazlik and M. Kara, "On a solvable system of difference equations of higher-order with period two coefficients," Communications Faculty of Sciences University of Ankara Series A1 Mathematics and Statistics, vol. 68, no. 2, pp. 1675-1693, 2019, doi: 10.31801/cfsuasmas.548262.

Authors' addresses

Y. Akrour

Youssouf Akrour, LMAM laboratory, Department of Mathematics, University of Mohamed Seddik Ben Yahia, Jijel, and Departement des Sciences Exactes et d'Informatique, École Normale Supérieure, Constantine, Algeria.

E-mail address: youssouf.akrour@gmail.com

\section{Kara}

Merve Kara, Department of Mathematics, Kamil Ozdag Science Faculty, Karamanoglu Mehmetbey University, Karaman, Turkey

E-mail address: mervekara@kmu.edu.tr

N. Touafek

(Corresponding author) Nouressadat Touafek, LMAM Laboratory, Department of Mathematics, University of Mohamed Seddik Ben Yahia, Jijel, Algeria.

E-mail address: ntouafek@gmail.com

\section{Y. Yazlik}

Yasin Yazlik, Department of Mathematics, Faculty of Science and Art, Nevsehir Haci Bektas Veli University, Nevsehir, Turkey

E-mail address: yyazlik@nevsehir.edu.tr 